\title{
Impact of deamidation on the structure and function of anti-apoptotic Bcl- $\mathbf{x}_{\mathrm{L}}$.
}

\author{
Gamze Tanriver, ${ }^{\mathrm{a}, \mathrm{b}}$ Gerald Monard ${ }^{\mathrm{b},{ }^{*}}$ and Saron Catak ${ }^{\mathrm{a},{ }^{*}}$ \\ aBogazici University, Department of Chemistry, Bebek, 34342 Istanbul, Turkey. \\ bUniversité de Lorraine, LPCT UMR 7019 CNRS, Boulevard des Aiguillettes B.P. 70239, 54506 Vandoeuvre-les-Nancy.
}

\begin{abstract}
$\mathrm{Bcl}-\mathrm{x}_{\mathrm{L}}$ is an anti-apoptotic mitochondrial trans-membrane protein, known to play a crucial role in the survival of tumor cells. The deamidation of $\mathrm{BCl}-\mathrm{x}_{\mathrm{L}}$ is a pivotal switch that regulates its biological function. The potential impact of deamidation on the structure and dynamics of $\mathrm{BCl}-\mathrm{XL}_{\mathrm{L}}$ is directly linked to the intrinsically disordered region (IDR), which is the main site for post-translational modifications (PTMs). In this study, we explored deamidation-induced conformational changes in Bcl$x_{L}$ to gain insight into its loss of function by performing microsecond-long molecular dynamics (MD) simulations. MD simulation outcomes showed that the IDR motion and interaction patterns have changed notably upon deamidation. Principal component analysis (PCA) demonstrates significant differences between wild type and deamidated $\mathrm{BCl}-\mathrm{x}_{\mathrm{L}}$ and suggests that deamidation affects the structure and dynamics of $\mathrm{BCl}-\mathrm{X}_{\mathrm{L}}$. Differences in contact patterns and essential dynamics in the binding groove (BG) are clear indications of deamidation-induced allosteric affects. Additionally, the importance of multiple simulations as well as long simulation time to examine structural changes from a broader perspective is emphasized. In line with previous studies, we show that the intrinsically disordered region plays a very important role in the loss of apoptotic function of $\mathrm{Bcl}-\mathrm{x}_{\mathrm{L}}$, while providing a unique perspective on the underlying mechanism of $\mathrm{BCl}-\mathrm{x}_{\mathrm{L}}$ deamidation-induced cell death.
\end{abstract}

\section{Introduction}

Bcl-2 family proteins (B-cell lymphoma-2), in combination with the mitochondrial outer membrane (MOM), control the fate of cells by regulating the mitochondrial pathway of apoptosis (programmed cell death). ${ }^{1-4}$ The $\mathrm{Bcl}-2$ family includes pro-apoptotic (BAX, BAK, and $\mathrm{BOK}$ ), anti-apoptotic ( $\mathrm{BCl}-2, \mathrm{Bcl}-\mathrm{X} \mathrm{L}, \mathrm{BCl}-\mathrm{W}$ and $\mathrm{MCL}-1)$ and $\mathrm{BH}$-only proteins (BOP), such as BIM, BID, PUMA, NOXA, and mediates the mitochondrial (intrinsic) apoptotic pathway in response to various apoptotic stimuli (cellular stress or damage signals). Bcl-2 family proteins maintain/preserve balance in healthy cells. When this balance is disrupted, elevated numbers of anti-apoptotic proteins are observed in various cancer cells, such as chronic myelogenous leukemia $(\mathrm{CML})$, pancreatic cancer, ovarian and small-cell lung cancer. ${ }^{5-7}$

Post-translational modification (PTM) is a regulatory mechanism in many biological processes. Most common modifications are acetylation, acylation, amidation, deamidation, phosphorylation, glycosylation, ubiquitination, nitrosylation and SUMOylation. ${ }^{8-10}$ Among PTMs, deamidation is of particular interest to this study. Deamidation is a chemical reaction that spontaneously occurs in proteins with the potential to substantially modify its structure and compromise its function. ${ }^{11,12}$ Asparagine (Asn) and glutamine (Gln), two of the 20 
naturally occurring amino acids, are inherently unstable under physiological conditions. ${ }^{12} \mathrm{Gln}$ and Asn are known to spontaneously yet non-enzymatically deamidate into a mixture of glutamyl (Glu) and iso-glutamyl (iso-Glu) and a mixture of aspartyl (Asp) and iso-aspartyl (isoAsp) residues, respectively (Scheme 1). ${ }^{13,14}$ This, in turn, replaces a neutral residue with a negatively charged one and has the potential to cause severe electrostatic clashes leading to structural deformations, which may eventually have dramatic biological consequences.

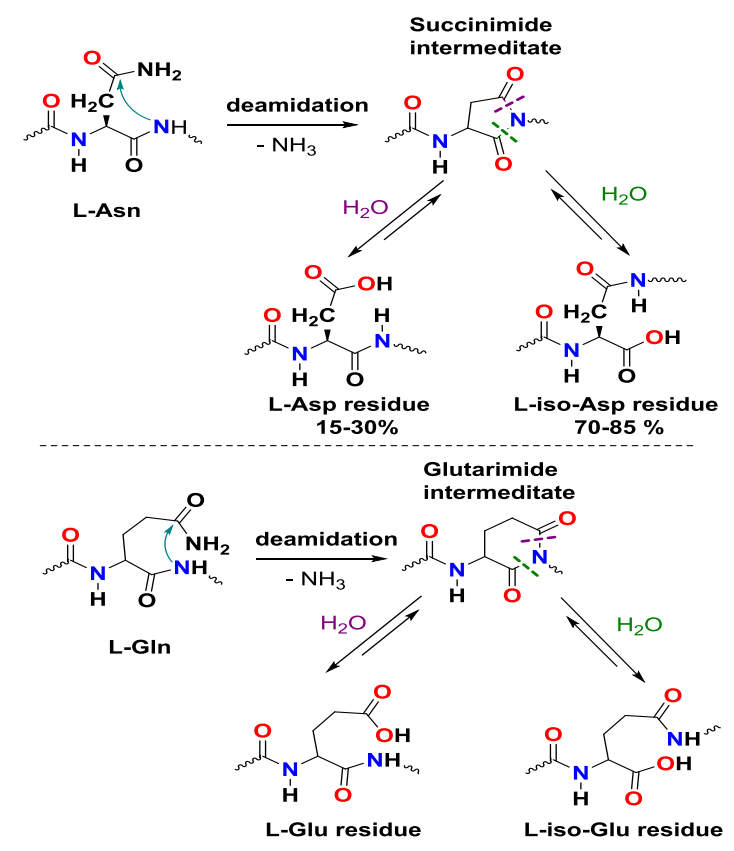

Scheme 1. General mechanism of Asn and Gln deamidation. ${ }^{15}$

Deamidation rate is determined by protein structure and environment; deamidation half times of proteins are shown to vary from a few hours to more than 100 years. ${ }^{11}$ Gln deamidation is usually substantially slower than Asn deamidation, hence asparagine deamidation has more biologically relevant consequences. A notable example is the mitochondrial transmembrane protein, B-cell lymphoma-extra-large $\left(\mathrm{Bcl}-\mathrm{x}_{\mathrm{L}}\right),{ }^{16,17}$ which functions as an anti-apoptotic protein. Bcl- $\mathrm{x}_{\mathrm{L}}$ belongs to the $\mathrm{Bcl}-2 \mathrm{family},{ }^{4}$ which regulates the mitochondrial pathway of apoptosis in response to various apoptotic stimuli. ${ }^{18} \mathrm{Bcl}-\mathrm{X}_{\mathrm{L}}$ indirectly binds to $\mathrm{BH} 3-o n l y$ proteins and prevents them from activating pro-apoptotic proteins or directly binds to pro-apoptotic proteins and inhibits their pro-apoptotic activities, which lead to cell death. ${ }^{19-21}$ Additionally, $\mathrm{Bcl}-\mathrm{x}_{\mathrm{L}}$ also indirectly plays a major role in autophagy $\mathrm{y}^{22,23}$ and senescence ${ }^{24,25}$ pathways. Since $\mathrm{BCl}-\mathrm{x}_{\mathrm{L}}$ is known to play a pivotal role in the survival of tumor cells, ${ }^{26}$ understanding the inter-relations among apoptosis, autophagy and senescence is pertinent in determining how Bcl-xL regulates cell survival or cell death.

Structurally, Bcl-X consists of two hydrophobic, six amphiphilic helices (Fig. 1) and a C-tail composed of an $\alpha$-helix ( $\alpha 9)$, known as the transmembrane domain (TMD). ${ }^{16,17,27,28}$ Note that to date, the C-tail ( $\alpha 9$ helix) had not been resolved in any of the available PDB structures of $\mathrm{BCl}-\mathrm{X}_{\mathrm{L}}{ }^{28}$ but more recently the $\mathrm{C}$-tail alone was experimentally resolved in phospholipid nanodiscs (PDB ID: 6F46 ${ }_{\mathrm{NMR}}{ }^{29}$ and PDB ID: 6 X7/ $\mathrm{NMR}_{\mathrm{NM}}$ ). Bcl-XL also comprises four $\mathrm{Bcl}-2$ homology domains (BH1-4) and a hydrophobic "binding groove" between $\alpha 2-\alpha 5$ helices capped by a 
short C-terminal ( $\alpha 8$ helix). The binding groove of $\mathrm{BCl}-\mathrm{X}_{\mathrm{L}}$ accommodates the $\mathrm{BH} 3$ domain of the BOP and pro-apoptotic proteins. Furthermore, $\mathrm{BCl}-\mathrm{x}_{\mathrm{L}}$ has an intrinsically disordered region (IDR) (residues 21-84), the so-called "loop region" between $\alpha 1-\alpha 2$. Specific residues within the IDR of $\mathrm{BCl}-\mathrm{X}_{\mathrm{L}}$ are post-translationally modified in response to diverse stimuli. Most of the previous studies lacked/omitted the IDR, ${ }^{30-34}$ however, the deletion of IDR increases the antiapoptotic activity of $\mathrm{BCl}-\mathrm{XL}_{\mathrm{L}}$ (hyperfunction), ${ }^{35}$ hence, $\mathrm{Bcl}-\mathrm{XL}_{\mathrm{L}}$ without IDR fails to give full insight into the structure and function of the protein. Analysis of the intrinsically disordered region is essential since the IDR is the main site for post-translational modifications (PTMs). ${ }^{36-39}$ The importance of the IDR as well as hotspots on the IDR have been reported in recent studies. ${ }^{40-}$ 44 Two asparagine residues (Asn52 and Asn66) ${ }^{41}$ on the IDR are well documented to deamidate and this, in turn, is known to play a pivotal role on reduction/loss of antiapoptotic activity of $\mathrm{BCl}-\mathrm{XL}_{\mathrm{L}}{ }^{40-42}$ The impact of PTMs on $\mathrm{BCl}-\mathrm{XL}_{\mathrm{L}}$ are not yet completely understood, however, apoptotic stimuli (DNA damage, UV, nutrient deprivation, chemotherapy, etc.) is known to induce PTMs in Bcl-XL that lead to $a$ ) the release of BOP and the subsequent activation of pro-apoptotic proteins, and $b$ ) the inhibition of its binding to pro-apoptotic and BH3-only proteins, leading to apoptotic cell death. ${ }^{2,21}$ One of the goals of this study is to elucidate the impact of PTMs that take place on the IDR, specifically deamidation, on the general structure of $\mathrm{Bcl}-\mathrm{x}_{\mathrm{L}}$, which may eventually lead to the release of BOPs or an inefficiency of sequestering BOPs.

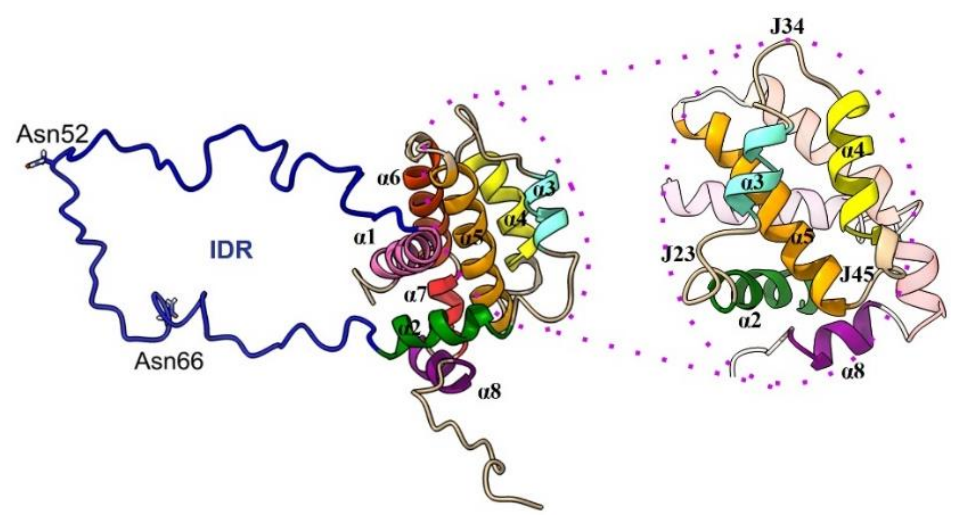

Fig. 1. Front and side views of $\mathrm{BCl}-\mathrm{xL}$ (PDB code: $1 \mathrm{LXL}$ ). (Purple dotted area indicates the binding groove (BG). Blue color represents loop region (IDR), $\alpha 1$ in pink, $\alpha 2$ in green, $\alpha 3$ in cyan, $\alpha 4$ in yellow, $\alpha 5$ in light orange, $\alpha 6$ in orange, $\alpha 7$ in red, and $\alpha 8$ in purple. "J" refers to the junction between two helices. J23 connects $\alpha 2$ and $\alpha 3, \mathrm{~J} 34$ is the junction of $\alpha 3$ and $\alpha 4$ and $\mathrm{J} 45$ connects $\alpha 4$ and $\alpha 5$.

Only three asparagine residues (Asn52, Asn66 and Asn185) with an ( $n+1)$ glycine residue (AsnGly) exist in structure of Bcl-XL. However, Asn185, being embedded in the core region, is not prone to deamidation due to less water-exposure; as a result, it has a much longer deamidation half-life when compared to Asn52 and Asn66. 6,16,45 Both asparagine residues, Asn52 and Asn66, are located in the IDR and undergo deamidation leading to a complete loss of protein function. $6,7,16,45,46$ While $\mathrm{Bcl}-\mathrm{X}_{\mathrm{L}}$ deamidation leads to apoptosis in normal cells, tumor cells are known to have acquired resistance to apoptosis and a clear survival advantage by suppressing $\mathrm{Bcl}-\mathrm{x}_{\mathrm{L}}$ deamidation and its biological consequences. ${ }^{6}$ In 1997, Aritomi et al. 
published the first report on $\mathrm{Bcl}-\mathrm{X}_{\mathrm{L}}$ 's susceptibility to deamidation, when defining the crystal structure of rat $\mathrm{Bcl}-\mathrm{X}_{\mathrm{L}}$ (PDB ID: $1 \mathrm{AF} 3_{\mathrm{X} \text {-Ray }}$. ${ }^{16}$ They defined the deamidation sites of rat $\mathrm{Bcl}-\mathrm{X}_{\mathrm{L}}$ by high performance liquid chromatography (HPLC) and iso-Asp was only detected in Asn52 and Asn66 showing the susceptibility of both residues. Similarly, Takehara and Takahashi reported that Asn52 and Asn66 residues of human $\mathrm{BCl}-\mathrm{X}_{\mathrm{L}}$ are prone to deamidation. ${ }^{6}$ In general, $\mathrm{Bcl}-\mathrm{X}_{\mathrm{L}}$ deamidation is accelerated by DNA-damage inducing agents used in cancer treatment. ${ }^{7,46,47}$ Notably, unlike the rest of the Bcl-2 family, asparagine-glycine (Asn-Gly) sequences in the IDR of $\mathrm{BCl}-\mathrm{X}_{\mathrm{L}}$ are evolutionarily conserved, showing their biological significance. ${ }^{47,48}$

Previously, Deverman et al. (Weintraub lab) reported that the deamidation of $\mathrm{Bcl}-\mathrm{x}_{\mathrm{L}}$ downregulates the anti-apoptotic activity of $\mathrm{BCl}-\mathrm{x}_{\mathrm{L} .}{ }^{45}$ However, in 2003 they published an erratum indicating that an undetected mutation was observed and $\mathrm{BCl}-\mathrm{XL}_{\mathrm{L}}-\mathrm{BIM}$ binding was recovered, that is, $\mathrm{BCl}-\mathrm{X}_{\mathrm{L}}$ does not lose its ability to bind to the $\mathrm{BH} 3$-only proteins upon deamidation. ${ }^{49}$ However, they had failed to consider the possibility of iso-Asp conversion in their study. Later, Alexander and coworkers confirmed the initial results of Deverman et al. by showing the loss of Bcl-XL's anti-apoptotic function when deamidated, and reported that iso-Asp52/iso-Asp66 residues in the loop region prevent the sequestering of $\mathrm{BH} 3$-only proteins. ${ }^{46}$ Therefore, it is imperative to further investigate the structural changes in $\mathrm{Bcl}-\mathrm{XL}_{\mathrm{L}}$ upon deamidation of Asn52 and Asn66 in order to clarify these seemingly controversial experimental results. The current study is the first step towards this goal, where we computationally explore the structural and biological consequences of $\mathrm{BCl}-\mathrm{X}_{\mathrm{L}}$ deamidation at the atomic level.

More recently, in 2018, Follis et al. reported a new NMR structure of a Bcl-xL phosphomimetic mutant (S62E-BCl-XL, PDB ID: 6BF2 $\mathrm{NMR}, 20$ conformers) and experimentally investigated the effect of PTMs in the intrinsically disordered region (phosphorylation and deamidation in IDR) on the regulation of apoptosis. ${ }^{40}$ They proposed that phosphorylation (S62E) and deamidation (N52D and N66D) in the IDR induce allosteric interactions in the binding groove leading to the release of pro-apoptotic proteins, which in turn trigger apoptosis.

Previous experimental studies, mentioned earlier, indicated the significant impact of deamidations in the IDR on the function of $\mathrm{BCl}-\mathrm{x}_{\mathrm{L}} \cdot{ }^{40-42,50-52}$ On the other hand, there is only a limited number of computational studies performed on Bcl- $x_{L}$ that includes the IDR. In 2013, Maity et al. reported significant differences between the conformational dynamics of $B c l-X_{L}$ in water and in membrane (total $1.6 \mu \mathrm{s}$, implicit models). ${ }^{32}$ In water, the C-tail covered the binding groove and acted as a lid. In water and membrane environments, principal components of the motions were significantly different implying conformational transitions of $\mathrm{BCl}-\mathrm{X}_{\mathrm{L}}$ in the membrane. However, the model used by Maity et al. was truncated by removing the Bak peptide from its complex with $\mathrm{Bcl}-\mathrm{XL}_{\mathrm{L}}$ (PDB ID: 1BXLNMR), moreover, the IDR was not present. In 2017 Priya et al. ${ }^{53}$ investigated the influence of the IDR on the function of $\mathrm{Bcl}-\mathrm{x}_{\mathrm{L}}$ by comparing $\mathrm{Bcl}-\mathrm{x}_{\mathrm{L}}$ with and without the IDR (total $800 \mathrm{~ns}$, NPT simulations). They showed that the IDR allosterically modulates the structural dynamics of $B C l-x_{L}$ and they validated this finding through testing the effect of phosphorylation of S49 and S62 in the IDR. Phosphorylation in the IDR induced changes in electrostatic interactions in the BG, showing allosteric regulation. In Priya's study, deamidation in the IDR was not explored and the complete experimental (NMR) structure (PDB ID: 1LXL (residues 2-196)) of Bcl-X was not utilized. More recently, in 2020, Marassi and coworkers described and investigated the 
conformation of full-length $\mathrm{Bcl}-\mathrm{XL}_{\mathrm{L}}$ in both its soluble and membrane-anchored states using NMR spectroscopy, isothermal titration calorimetry (ITC) and molecular dynamics ( $1 \mu \mathrm{s} \times 5 \mathrm{MD}$ simulations). ${ }^{54}$ The study mostly focused on the tail-groove interaction in solution. Their model was modified $\mathrm{Bcl}-\mathrm{x}_{\mathrm{L}} / \mathrm{Bak}-\mathrm{BH} 3$ complex (PDB: $1 \mathrm{BX} \mathrm{L}_{\mathrm{NMR}}$ ) by adding a loop section from $1 \mathrm{LX} \mathrm{L}_{N M R}$ and by replacing the C-terminal His-tag with the C-terminal tail (residues 210-233) from $6 \times 7 I_{\text {NMR. }}$. The Bak-BH3 peptide was removed, and the $\mathrm{C}$-tail was docked into the binding groove. The MD simulations in their study was somewhat limited and did not offer any insight on deamidation in the IDR. Taken together, the computational studies, in general, had short simulation times (maximum $1 \mu \mathrm{s}$ ) causing limited sampling; moreover, these studies used truncated or modified models of $B c l-x_{L}$, where the IDR was subsequently added. To date, the mechanism by which $\mathrm{Bcl}-\mathrm{x}_{\mathrm{L}}$ deamidation induces apoptosis remains unclear. In the current study, we used a non-modified, non-truncated experimentally available form of $\mathrm{Bcl}-\mathrm{xL}$, unlike previous studies. This study is the first attempt to investigate the structural and biological consequences of $\mathrm{BCl}-\mathrm{x}_{\mathrm{L}}$ deamidation at atomic resolution through microsecond long $\mathrm{MD}$ simulations. In this context, essential dynamics and $\mathrm{H}$-bond interactions were particularly scrutinized.

\section{Computational Methods}

\section{Simulation Details}

Initial structure "1LXL" was retrieved from the Protein Data Bank (Homo sapiens); cloning artifacts and histidine tags were removed. MD simulations were performed by using the Amber18 program package. ${ }^{55}$ All MD simulations were performed using the Amberff14SB ${ }^{56}$ force field implemented in the Amber18 program package ${ }^{55}$ and solvation was carried out using the TIP3P ${ }^{57}$ explicit water model forming cubic boxes. Counter ions (sodium or chloride) were added into each system to neutralize charges. Amber force field for L-iso-Asp, which is a non-standard amino acid, was parameterized with the antechamber and tleap modules as implemented in the Amber simulation package (see details in SI: Fig. S1 and Table S1). After the preparation step, all systems (WT, DM1 and DM2) were minimized using the steepest descent method with harmonic restraints on all heavy atoms. In the minimization step, NVT ensemble with Andersen temperature coupling ${ }^{58}$ was employed and the velocities were randomly updated every 10 steps. Long range interactions were calculated using the particle mesh Ewald (PME) ${ }^{59}$ method with the default $8 \AA$ cutoff distance. SHAKE algorithm was carried out in order to constrain bonds involving hydrogen atoms. ${ }^{60}$ Equilibration runs were performed using the NPT ensemble with a Monte Carlo barostat. Time step for the equilibration runs was set to $2 \mathrm{fs}$. A five-step equilibration protocol was conducted as follows: 1) all heavy atoms were restrained with a harmonic potential of $50 \mathrm{kcal} \mathrm{mol}^{-1} \AA^{-2}$ for $0.1 \mathrm{~ns}$ at $10 \mathrm{~K}$, to provide a proper geometry for the $\mathrm{H}$ atoms. 2) restraints on the oxygen atoms of water molecules were removed to optimize the positions of water molecules with respect to the protein environment using the same restraining potential for $0.1 \mathrm{~ns}$ at $10 \mathrm{~K}$. 3) the harmonic potential was decreased to $5 \mathrm{kcal} \mathrm{mol}^{-1} \AA^{-2}$ by repeating the 2 nd step. 4) the entire system was equilibrated without restraints for $0.1 \mathrm{~ns}$ at $10 \mathrm{~K}$. Under the Andersen thermostat ${ }^{58}$ the velocities were randomly updated every 10 steps throughout equilibration steps 1-4. 5) The systems were gradually heated to $300 \mathrm{~K}$ for $2 \mathrm{~ns}$ using the Berendsen 
thermostat ${ }^{61}$ with a coupling time of $1.0 \mathrm{ps}$. Production runs were performed at $300 \mathrm{~K}$ and an NVT ensemble utilizing Langevin temperature coupling ${ }^{62}$ with a gamma of $1.0 \mathrm{ps}^{-1}$. The time step for production runs was set to $4 \mathrm{fs}$, using hydrogen mass repartitioning $(\mathrm{HMR})^{63}$, to accelerate MD simulations.

\section{Analysis Details}

All analyses were performed with the cpptraj ${ }^{64}$ module of Amber18. The backbone root meansquare deviation (RMSD) analysis was performed using the backbone atoms of Bcl-x 's core region defined by residues 85-96, 120-131, 137-156, 162-176 in line with the core region definition (residues $85-98,123-127,140-156$, and 162-175) of Liu et al. ${ }^{30,31}$ Secondary structure contents were calculated using the Definition of Secondary Structure Prediction (DSSP) algorithm. ${ }^{65}$ For hydrogen bond (H-bond) calculations, the distance criteria was defined as $\leq 3.2 \AA$ based on heavy atom distances (acceptor to donor heavy atom). Contact percentage (\%) in $\mathrm{H}$-bond analysis is defined as the percentage of total contacts during simulations. Linear Interaction Energy (LIE) analysis was performed to evaluate non-bonded long-range interactions. The results were visualized by B-factor coloring to effortlessly pinpoint the hotspots between IDR and protein (excluding IDR, $\triangle I D R$ ).

Principal component analysis (PCA) was performed with the cpptraj module and principal components (PCs) were visualized with the Normal Mode Wizard (NMWiz) ${ }^{66}$ in VMD (1.9.3). Firstly, the combined trajectories were RMS-fitted to an overall averaged structure using the backbone atoms of the binding groove (residues 85-156) to remove global translational and rotational motions. The coordinate covariance matrices (288 atoms total, 864 coordinates) were calculated using the same mask, namely the binding groove (residues 85-156). Then, the coordinate covariance matrices were diagonalized to obtain the first 10 PCs (i.e., eigenvectors) and their eigenvalues. All histograms in PCA were calculated using a Gaussian kernel density estimator (KDE). Graphics were plotted by Gnuplot (version 4.6) (http://www.gnuplot.info/) and Xmgrace (version 4.0). Chimera ${ }^{67}$ (version 1.14), ChimeraX, ${ }^{68}$ and $\mathrm{VMD}^{69}$ (version 1.9.3) were used for the visualization and illustration of the studied models.

Table1. Summary of the Initial Set up ${ }^{a}$

\begin{tabular}{|c|c|c|c|c|}
\hline $\begin{array}{c}\text { Initial } \\
\text { Structure }\end{array}$ & Water & $\mathrm{Na}+$ & $\begin{array}{c}\text { Total } \\
\text { atoms }\end{array}$ & Simulation time \\
\hline $\begin{array}{c}\text { 1LXL }_{\text {NMR }} \\
\text { (Bcl-x } \\
\text { WT) }\end{array}$ & 44408 & 14 & 136431 & $5 \mu \mathrm{s} \times 4,9 \mu \mathrm{s} \times 1$ \\
\hline $\begin{array}{l}\text { DM1-1LXL } \\
\text { (DM1) }\end{array}$ & 44406 & 16 & 136423 & $5 \mu \mathrm{s} \times 4,10 \mu \mathrm{s} \times 1$ \\
\hline \multirow[t]{2}{*}{$\begin{array}{c}\text { DM2-1LXL } \\
\text { (DM2) }\end{array}$} & 44406 & 16 & 136423 & $5 \mu \mathrm{s} \times 3,8 \mu \mathrm{s} \times 2$ \\
\hline & & & Total & $90 \mu \mathrm{s}$ \\
\hline
\end{tabular}




\section{Results and discussion}

From a computational viewpoint, the identification of conformational differences plays a pivotal role in understanding the impact of deamidation in IDR. Up to now, there is no clear structural connection/explanation on how deamidation induces the loss of Bcl- $\mathrm{X}_{\mathrm{L}}$ activity. In order to scrutinize conformational behaviors/differences of wild-type (WT) and deamidated $\mathrm{Bcl}-\mathrm{X}_{\mathrm{L}}$ systems, microsecond-long MD simulations were performed using different starting velocities. The stability of the systems and $\mathrm{H}$-bond interactions were explored. Then, using PCA, we specifically focused on the conformational changes in the binding groove (BG) upon deamidation of IDR residues Asn52 and Asn66, by considering both Asp and iso-Asp mutations. The terms "DM1" and "DM2" refer to Asp and iso-Asp deamidated Bcl- $\mathrm{X}_{\mathrm{L}}$, respectively. An overview of all the systems studied is shown in Table 1. Overall, the total amount of MD simulation time is $90 \mu \mathrm{s}$.

\section{Stability of the wild type and deamidated Bcl-xL systems}

A backbone root mean-square deviation (RMSD) analysis was performed for wild type and deamidated $\mathrm{Bcl}-\mathrm{x}_{\mathrm{L}}$ 's core region and their binding grooves (residues 85-156) (Fig. 2) as well as the IDRs (residues 21-84) (Fig. 3) to evaluate the stabilities of the systems during the simulations. RMSD plots in Figs. 2 illustrate that the core regions of WT, DM1, and DM2-Bcl$\mathrm{X} \mathrm{L}$ are stable $(<2.0 \AA)$ during the simulations. However, the RMSD of the core region for the first simulation of DM1 (DM1-SIM1) is slightly higher $(<3.0 \AA$ ) than the others. Therefore, we extended the simulation up to $10 \mu$ s to see if the backbone RMSD would further increase and it remained within the 1.5-3.0 $\AA$ range. We accepted and further investigated this simulation in the following analyses. Per-residue RMSD analysis was also performed to see which helices in the core and the BG are responsible for the increase in RMSD of DM-SIM1 (Figs. S2-S3). Average per-residue RMSD analysis illustrates that $\alpha 2$ shows higher fluctuations. Additionally, regarding all simulations (WT, DM1, and DM2), the J23 junction connecting $\alpha 2$ and $\alpha 3$ displays larger deviation with higher per residue RMSD in DM1-SIM1 (Fig. S3).

Fig. 3 shows that the IDR is highly flexible during the simulations. It is noteworthy that the RMSD fluctuations are, on average, lower for the deamidated versions indicating that the loop may be less flexible due to increased interactions with the core. Next, we further look into specific interactions that may be causing this behavior.

We also evaluated the stability of the helices during the MD simulations through secondary structure (SS) analysis (Fig. 4). We saw that $\alpha 1-\alpha 8$ helices were generally preserved in all simulations. Interestingly, persistent (long-lived) and/or 'transient but re-occurring' helical formations in the loop were observed as shown in Fig. 4 (all SS plots in Figs. S4-S6).

\section{Intrinsically disordered region (IDR, loop)}

Literature studies have shown that understanding the influence of post-translational modifications, i.e., deamidation on the IDR will lead to better insight into regulation and downregulation of $\mathrm{BCl}-\mathrm{X}_{\mathrm{L} .}{ }^{40-42,70}$ Structurally, the initial extended form of the IDR was not 
preserved throughout the simulations. Instead, simulations show that the interactions between the IDR and the protein core region are transient (short-lived) and consistently reoccurring, and the IDR approaches the core region in various ways. Particularly to verify this observation we performed several simulations, and in each case the IDR did not stay extended and approached the protein core even though the simulation boxes were large enough to accommodate extended forms. Hence, it is safe to say that the 1LXL PDB structure does not constitute a representative structure of the IDR. The IDR generally moves towards the core regions and mostly interacts with $\alpha 1, \alpha 2$ and $\alpha 6$ throughout the simulations. Despite the large flexibility of the IDR, transient (short-lived) but consistently re-occurring interactions, exist between the loop and protein core, in all independent simulations.
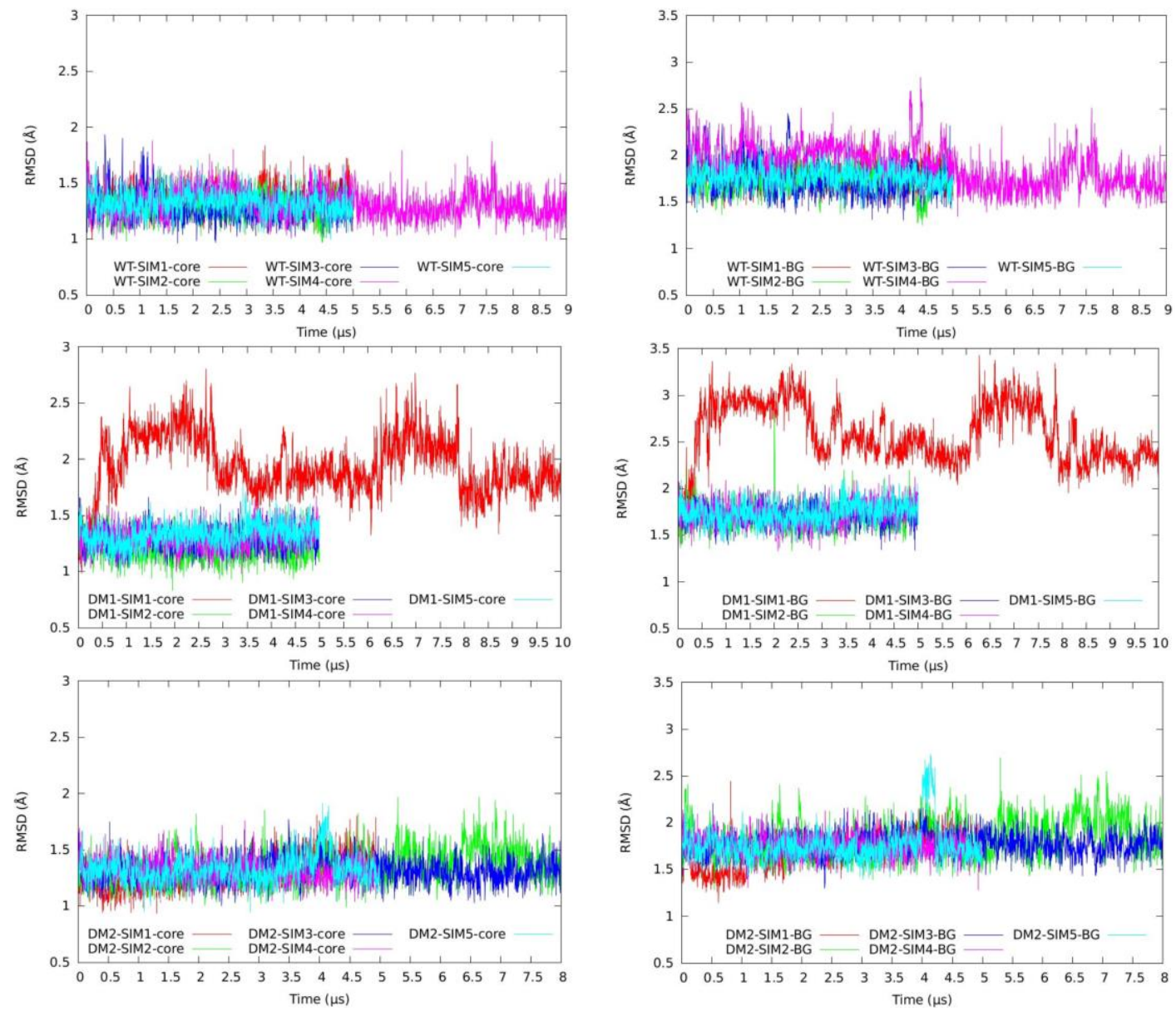

Fig. 2. Backbone RMSD plots for core helices (left) and binding groove (BG) (right) of wild type, DM1 and $\mathrm{DM} 2-\mathrm{BCl}-\mathrm{X}_{\mathrm{L}}$ simulations with respect to $1 \mathrm{LXL}_{\mathrm{NMR}}$.

Non-covalent interactions $(\mathrm{NCl})$, such as salt-bridges, $\mathrm{H}$-bonds, and cation- $\pi$ interactions are known to contribute to the stability of proteins. Among the $\mathrm{NCl}, \mathrm{H}$-bond interactions in particular were explored, and long-lived (persistent) interactions were distinguished from transient (short-lived) and consistently re-occurring interactions. Herein, attention will be drawn to short-lived interactions that re-occur in more than $30 \%$ of the total simulation time. IDR residues represented in bold throughout the manuscript. $\mathrm{H}$-bond interactions of IDR residues 52 (N52, D52, iso-D52) and 66 (N66, D66, iso-D66) with the protein core (excluding the loop) were examined before and after deamidation to check for disruption of pre-existing 
or formation of new interactions. Representative snapshots of $\mathrm{WT}, \mathrm{DM} 1$, and DM2-Bcl-X involving $\mathrm{H}$-bond interactions between IDR and core are shown in Fig. 5. In three out of the five wild type simulations, N52 interacts with the core residues of the protein. $\mathbf{N 5 2}$ interacts with R91 (23.3 \%) in the first simulation of the wild type (WT-SIM1), R6 (36.3 \%) and S164 (24.1 \%) in WT-SIM2 (Fig. 5a-b). N52 also transiently interacts with E202 (12.5 \%) and R204 (11.2\%) in WT-SIM4.

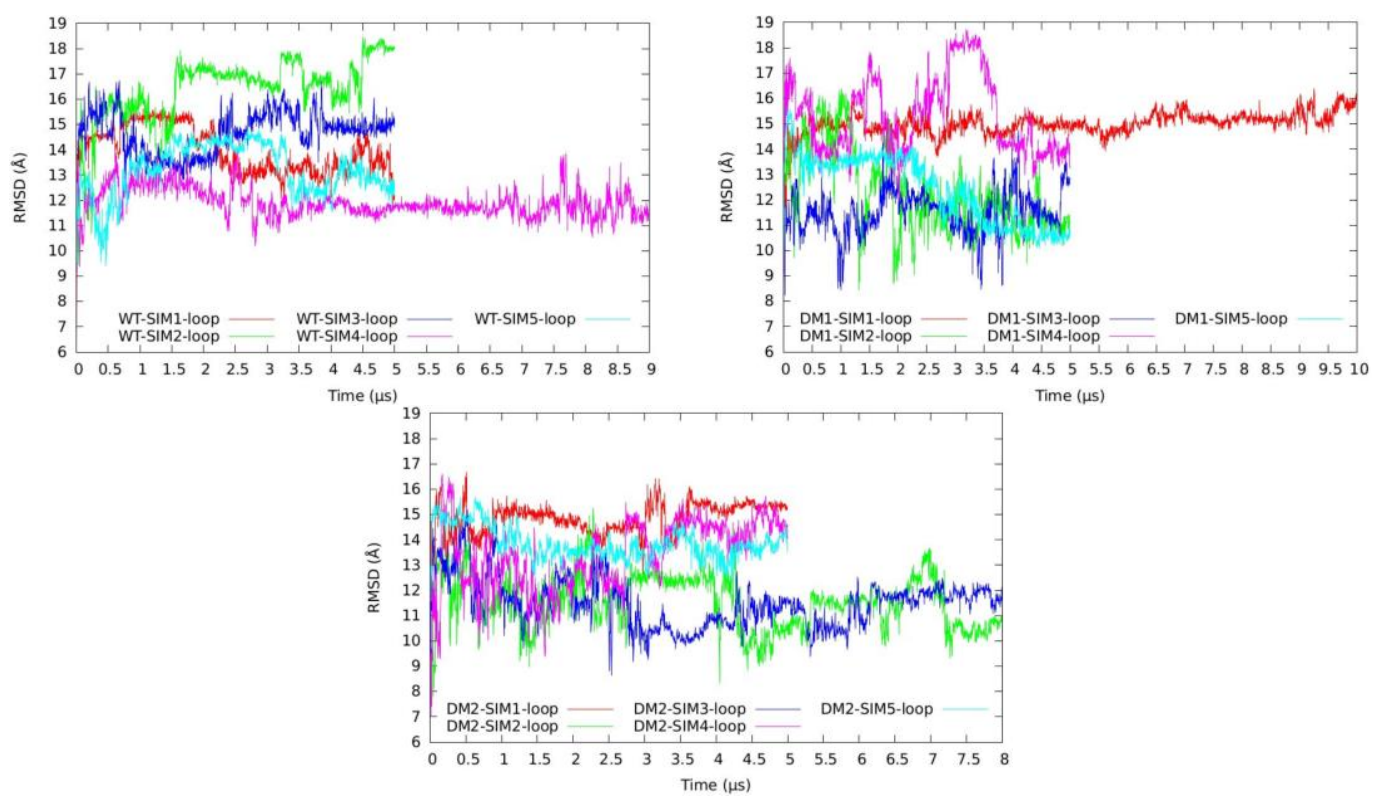

Fig. 3. Backbone RMSD plots for the IDR of WT, DM1 and DM2-Bcl- $x_{L}$ simulations with respect to initial structure.

More interestingly, once deamidated (simulation DM1), mostly D66 instead of D52, interacts with the core residues. In two out of the five simulations, D66 interacts with R91 (54.3\%), Y15 (25.7 \%) in DM1-SIM1 and Q88 (29.8\%) in DM1-SIM5, while N66 in wild type did not interact with neither Arg91, Tyr15 nor GIn88 (Fig. 5C-d). D52 also interacts with the first three residues (M1, S2, and Q3) (approximately $19 \%$ per residue) in DM1-SIM3. Compared to wild type and DM1, in DM2 (iso-Asp) simulations, $\mathrm{S} 2$ in $\alpha 1$ has transient interactions with iso-D52 in DM2SIM1 (13.5 \%) and with iso-D66 (19.2 \%) in DM2-SIM2 (Fig. 5d-e). As a result of the structural changes that iso-Asp deamidation introduces in the IDR backbone, the number and persistence of interactions between residues iso-D52 and iso-D66 and the protein core were considerably less. Linear Interaction Energy (LIE) analysis was also performed to evaluate long-range interactions between the protein core and the IDR. Average LIE analysis results (Fig. S7) also complied with the $\mathrm{H}$-bond analysis results mentioned above. Additionally, isoD52 in DM2-SIM3 and DM2-SIM4 and iso-D66 in DM2-SIM4 displayed stabilizing interactions with the protein core.

Interestingly, although non-existent in WT simulations, persistent $\mathrm{H}$-bond interactions between IDR residue $\mathbf{S 7 4}$ and a multitude of residues in the protein core were observed in each independent simulation of DM2. S74 interacts with D11 (88.6\%) and K87 (81.3\%) distinctively in DM2-SIM1, with D189 (72.8\%), Q3 (47.7\%) and E179 (88.6\%), respectively in 
DM2-SIM2, DM2-SIM3, and DM2-SIM-4. Moreover, the $\mathrm{H}$-bond interaction profile of core residue R91 changed significantly. R91-N52 (23.3\%) and R91-E46 (63.9\%) interactions were originally observed in WT-SIM1 and WT-SIM4, respectively. However, consequent to deamidation, R91-D66 (54.3 \%), R91-E42 (51.2 \%) and R91-A60 (50.5 \%) in DM1 (SIMs 1, 4 and 5 ) and R91-H71 (86.3 \%) in DM2-SIM1 were detected. These findings indicate that the loop behavior has changed significantly upon deamidation; this will be further analyzed in the following sections.

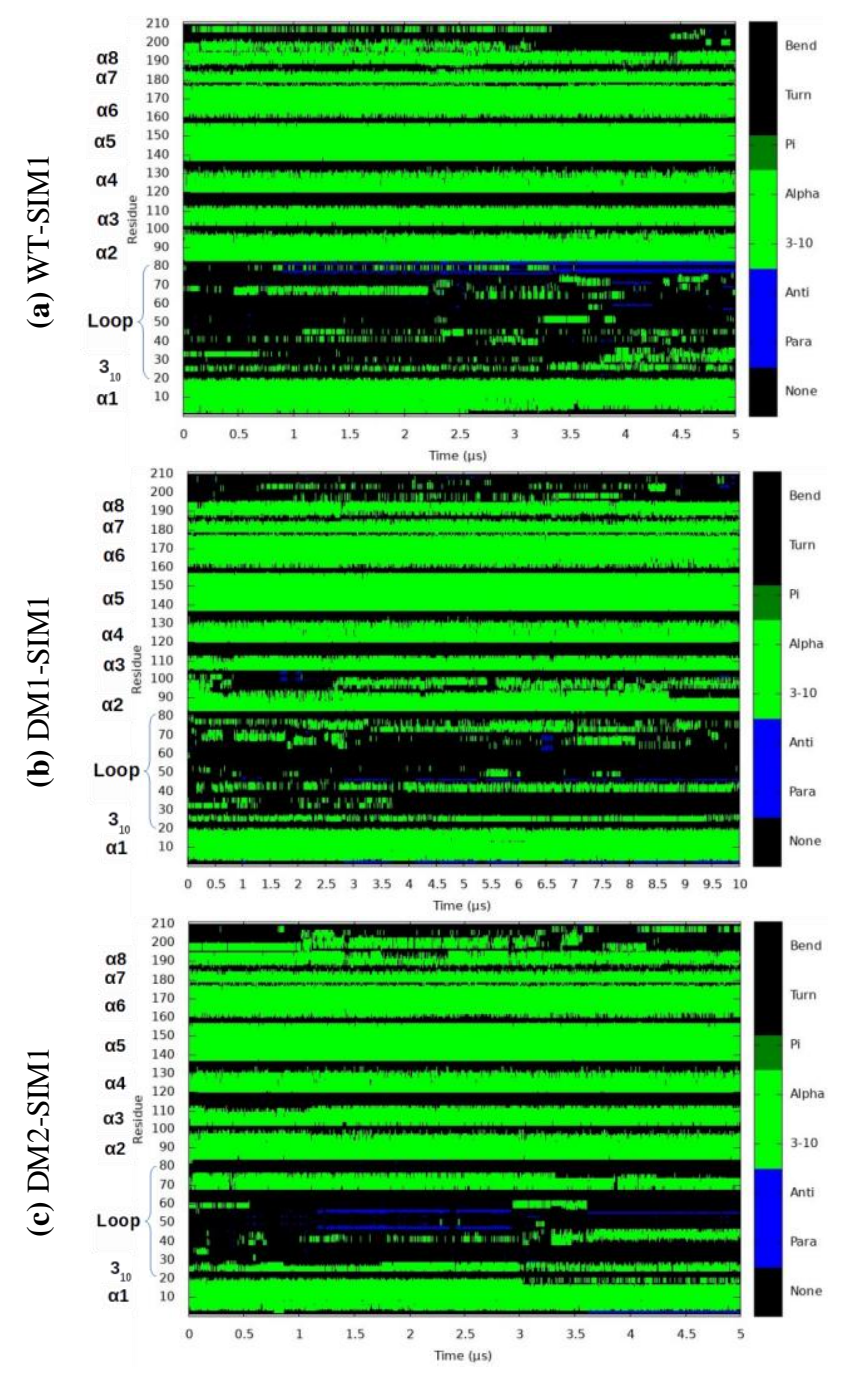

Fig. 4. Secondary structure evolution for the selected WT, DM1, and DM2 simulations. (Green color presents $\alpha$ helix and $3_{10}$ helix structures during the simulations.)

Additionally, the IDR interacts with itself (intra-loop regions) and persistent hinge formations were observed. As expected, all independent simulations showed different interaction patterns. This outcome supports the flexibility of the IDR. Taken together, these results highlight the diversity of the loop-protein core interactions. The extra negative charge introduced via deamidation understandably induces changes in the $\mathrm{H}$-bond interaction patterns between the IDR and the protein core. These interactions could allosterically affect the dynamics of Bcl-XL. Accordingly, essential dynamics of WT and deamidated systems will be comparatively explored in the following section. 
Priya et al. ${ }^{53}$ in their computational study (100 $\mathrm{ns} \times 2$ in total for $\mathrm{Bcl}-\mathrm{X}_{\mathrm{L}}$ with IDR) had reported transient interactions between the IDR and the core region of the $\mathrm{Bcl}-\mathrm{x}_{\mathrm{L}}$. In line with the findings of Priya et al., most of our simulations for WT and DM1 show that the loop residues E31, E32, E39 and $\mathbf{R 7 8}$ temporarily interact with core residues R165, R6, R91, and E7, respectively. All interactions mentioned above are also observed in DM2 simulations, except for the E39-R91 interaction. On the other hand, more persistent interactions between E31R165 in DM1-SIM2 and DM1-SIM3 and between R78-E7 in DM1-SIM4 and WT-SIM5 are observed in more than $40 \%$ of the MD simulations. Our simulations verified the transient but re-occurring nature of these interactions. This, once again, is an indication that the contact patterns in the reported NMR structure are different than the simulated ones.

In DM2 simulations, the additional carbon atom added to the IDR backbone upon conversion of Asn to iso-Asp is expected to cause substantially larger conformational changes compared to those caused by conversion to Asp. Intriguingly, loop movements in DM2-SIM4 and DM2SIM5 were considerably different than all other simulations (Figs. 6a and 6c). Figs. 6a and 6c depict mid-loop residues approaching the binding groove (BG), more specifically, $\alpha 4$ and the junction of $\alpha 4$ and $\alpha 5$ (J45) in DM2-SIM4 and the junction of $\alpha 3$ and $\alpha 4$ (J34) in DM2-SIM5. Additionally, transient interactions (E124-W57, N128-S62, R132-D61) between IDR and BG were seen in DM2-SIM4 (Fig. 6b). In DM2-SIM5, short-lived P116-S43 and Q121-A37 interactions were also observed as demonstrated in Fig. 6d. DM2 simulations suggest that the extra carbons in the backbone, caused by Asn conversion to iso-D52 and iso-D66, change the conformational behavior of the IDR, as seen by the loop's approach to the BG; this may prevent/affect binding ability of BOP or pro-apoptotic proteins to the binding groove of $\mathrm{Bcl}$ $\mathrm{X} L$.

Furthermore, in the experimental work of Follis et al. in 2018, negatively charged D61, E62, D52, and D66 in the loop region were positioned near and electrostatically interacted with the positively charged arginines (R100, R102, R103; known as the 'Arginine cluster') on the J23 of the BG. However, in the experimental NMR model reported by Muchmore et al. ${ }^{17}$ (PDB ID: $1 \mathrm{LXL}$ ), neither S62 and D61 nor $\mathbf{N 5 2}$ and $\mathbf{N 6 6}$ interacted with the Arginine cluster in J23. In the current study, we monitored interactions between deamidation residues 52 (N52, D52, and iso-D52) and 66 (N66, D66, and isoD66) and the Arginine cluster in all systems (WT, DM1, and DM2). Radial distribution functions (RDF) between the Arginine cluster's guanidinium hydrogens and the side chain oxygen atoms of residues 52 and 66 were calculated to monitor these interactions. The RDF plots show no interaction in the range of $0-10 \AA$ in 13 out of 15 simulations (Fig. S8). However, in two simulations (WT-SIM4 and D1SIM4), RDF showed interactions in the range of 3.0-6.0 $\AA$. To check the viability of these interactions, distance analysis was performed on simulations WT-SIM4 and D1-SIM4. Distance plots depict that these interactions are not persistent during the simulations (Figs. 7a and 7b).

More importantly, Follis et al. claimed that PTMs (phosphorylation and deamidation) that lead to these interactions induce a structural rearrangement at the distal binding groove (BG), which could lower Bcl-XL's affinity for BOP. Therefore, we focused on possible structural rearrangements in the binding groove, which stem from deamidation, and affect the dynamics of the distal BG. To do so we have performed thorough Principal Components Analysis as shown in following sections. 


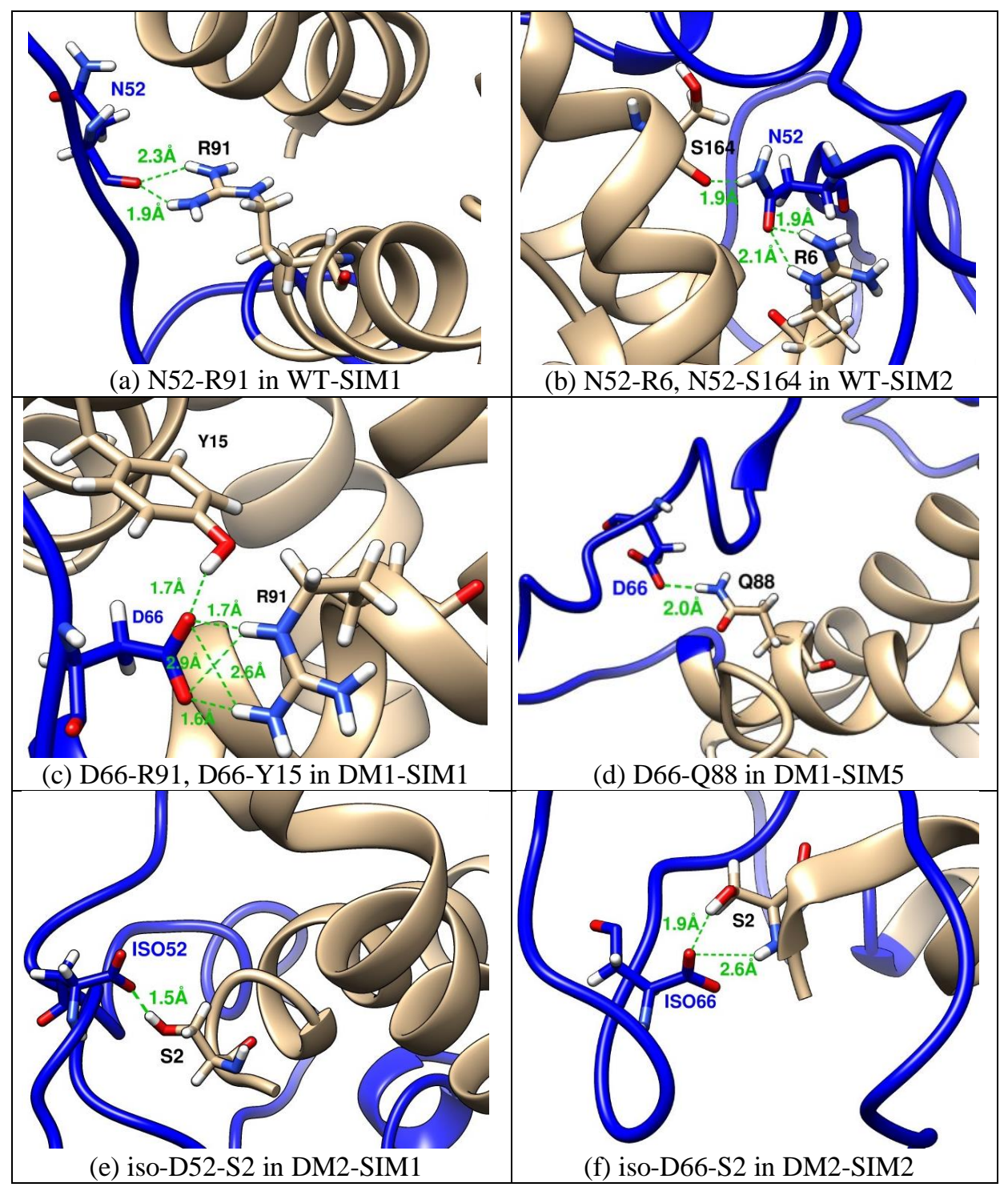

Fig. 5. $\mathrm{H}$-bond interactions between residue 52 , residue 66 , and protein core in representative WT, DM1, and DM2-Bcl-x simulations. Blue color denotes loop region (IDR); ISO52 and ISO66 refer to iso-Asp52 and iso-Asp66, respectively. 

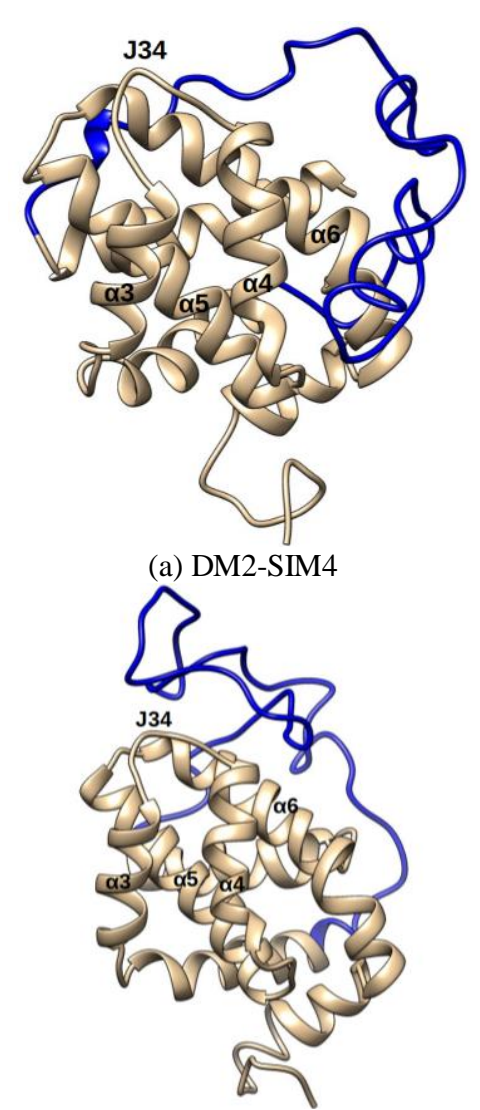

(c) DM2-SIM5

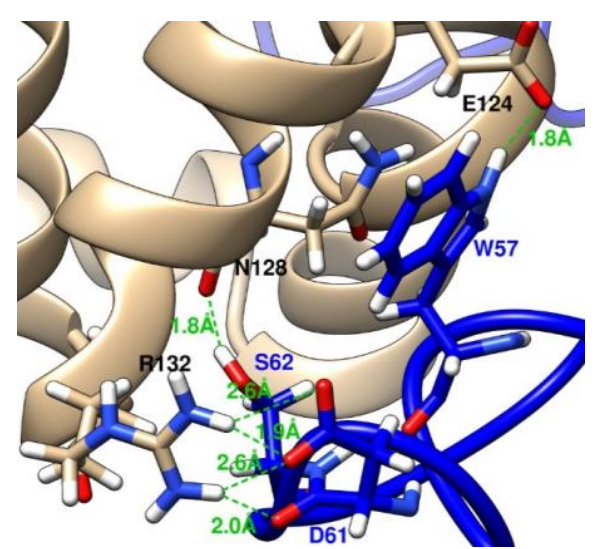

(b) W57-E124, S62-N128, D61-R132 in DM2-SIM4

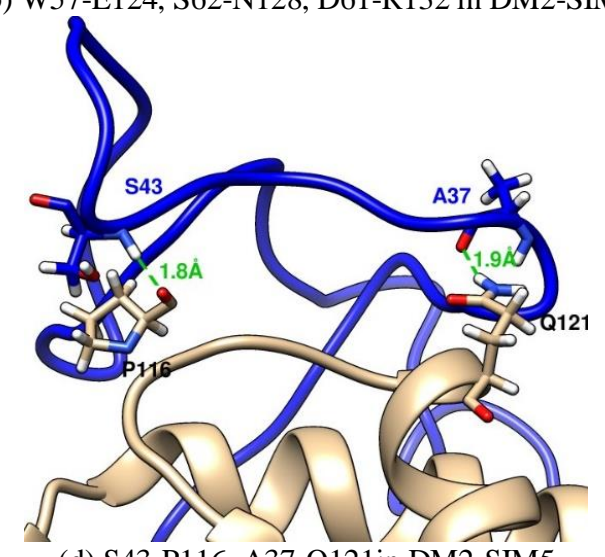

(d) S43-P116, A37-Q121in DM2-SIM5

Fig. 6. a, c) Representative positions of the loop. b, d) some representative transient interactions between loop and $\alpha 4, \mathrm{~J} 45$ and J34 for DM2-SIM4 and DM2-SIM5. (Blue color denotes the loop.)
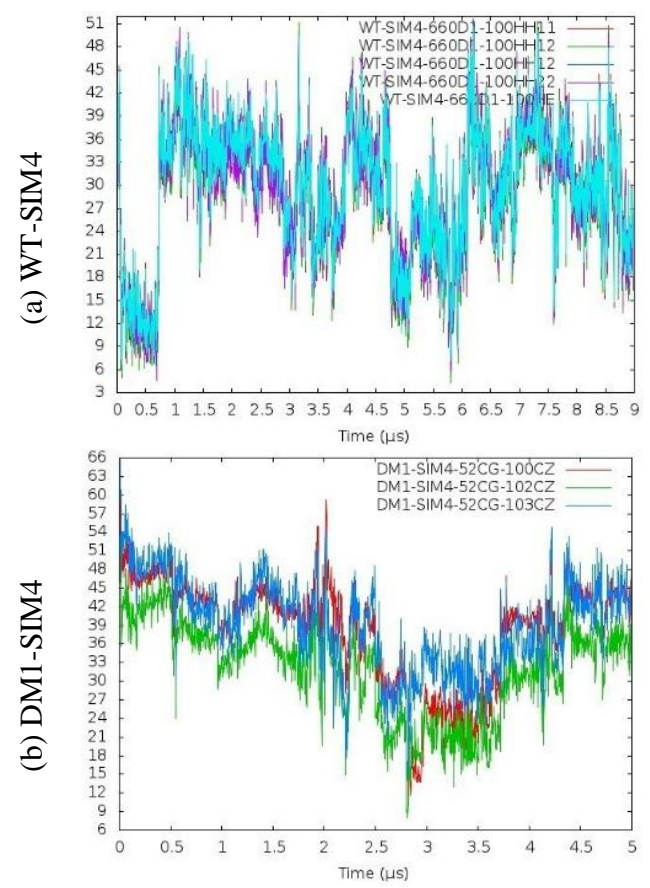

Fig. 7. Distance plot between a) N66@OD1 and guanidinium H atoms of R100. b) D52@CG and R100,102,103@CZ. 


\section{Principal Components Analysis (PCA)}

PCA provides insight into the dominant motions and the essential dynamics of the system. ${ }^{71}$ Briefly, PCA translates Cartesian coordinates (trajectories) into the dominant motions. Through PCA, the conformational differences between simulations are explored by investigating the distribution overlap; visual inspection provides information on the similarity of the motions. Since the binding groove which accommodates the BOPs and pro-apoptotic proteins is the main/crucial site for the execution of the anti-apoptotic function of $\mathrm{BCl}-\mathrm{X}_{\mathrm{L}}$, we focused on the evaluation of the BG dynamics. The structural differences induced by deamidation were further investigated by comparing the essential dynamics of the systems studied. PCA computations were performed in two steps: a) by combining all independent simulations (15 in total) and by identifying each system (WT, DM1, and DM2) that is particularly different from the majority b) by visually comparing the PC modes of the selected simulations against the reference WT system (ref-WT). (Further details in Computational Methodology under Analysis details.)

First, 15 independent simulations were combined and each system (WT, DM1 and DM2) was separately projected. We limited our analysis to the first four PCs, since approximately $80 \%$ of the cumulative variance was covered by them (Fig. 8b). For DM1, about $80 \%$ of the motions were described by the first three eigenvectors only, while a fifth and even a sixth eigenvector is needed for WT and DM2, respectively, to reach $\sim 80 \%$ of the cumulative variance, implying a higher flexibility for the latter systems. PC projection histograms in Fig. 9 illustrate that PC1 and PC2 show significant differences among the systems (WT, DM1 and DM2), particularly for DM1 indicating considerable conformational changes. These results also imply the difference in overall motions among the systems. PC scatter plots in Fig. $\mathbf{1 0}$ showed distribution of conformations along the first three PCs. DM1 scans different and larger conformational spaces as depicted in Fig. 10. Large conformational sampling in DM1 refers to notable conformational changes. The first two PCs in DM1 overlap less when compared to WT and have two distinct conformational subspaces. In addition, a portion of PCs in DM1 overlap with the conformational subspace of DM2. To understand which simulations cause these differences, each was separately projected onto the combined trajectories (Figs. S9-S11).

For WT, simulations 3 and 4 (WT-SIM3 and WT-SIM4) overlap less with the rest of the WT simulations (Fig. S9). Particularly the first PC of WT-SIM4 is considerably different with respect to other WT simulations. Moreover, PC scatter plots display that WT-SIM3 covers a similar space with WT-SIM1, WT-SIM2 and WT-SIM5 indicating that similar types of motions were sampled (Fig. 11). WT-SIM4 scans larger conformational spaces but overlaps with the rest of the WTs and does not overlap with DM1 simulations, particularly not with D1-SIM1 (data not presented here, see Fig. S12). Pertaining to DM1, simulations 1 and 2 (DM1-SIM1 and DM1SIM2) stand out with respect to the rest of the DM1s (Figs. 11 and S10). For DM2, simulation 2 (DM2-SIM2) overlaps less with the rest of the DM2 simulations (Figs. S11) and covers considerably larger conformational space. DM2-SIM1 and DM2-SIM5 also span slightly different subspaces when compared to the rest of the DM2s (Figs. S11 and 11). 


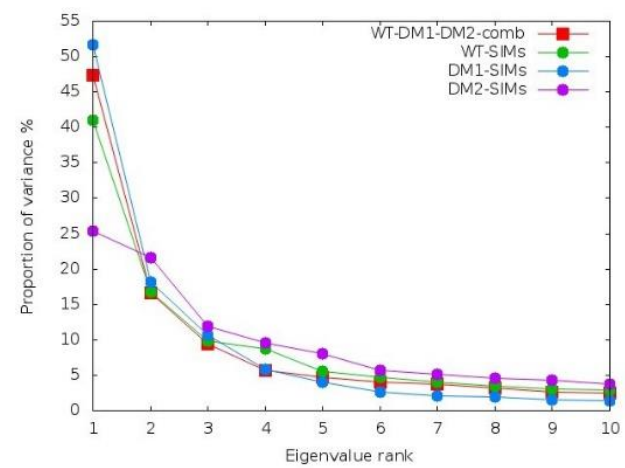

a) Proportion of variance

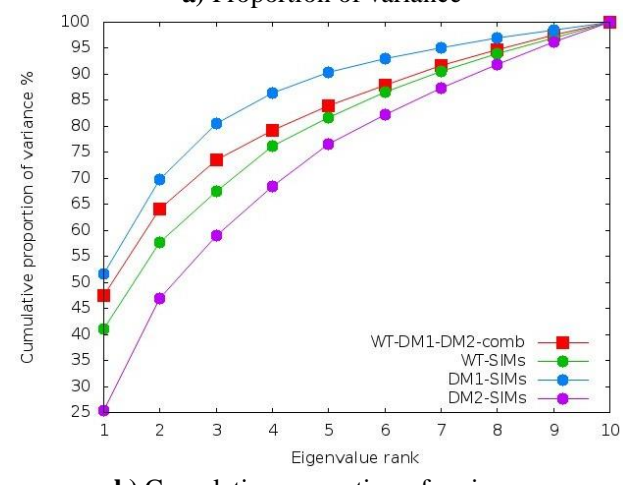

b) Cumulative proportion of variance

Fig. 8. a) Proportion of variance b) Cumulative sum of total variance captured by the first 10 PCs for the combined MD runs (WT+DM1+DM2-comb) and separately combined WT, DM1 and DM2 simulations.

Secondly, three of the similar WT simulations (WT-SIM1, WT-SIM2 and WT-SIM5) were combined in order to establish a reference for WT simulation. This was then used as a reference (ref-WT). The three normal modes of this reference system were compared visually with other simulations that showed significant differences. Since the first three PCs generally cover the overall motion as verified earlier with PC plots, the first three PC motions (modes) were compared by using NMWiz ${ }^{66}$ in VMD (1.9.3) (Fig. 12, Figs. S13-S15). The most prominent motion in the PCs of DM1 is the mobility of the $\alpha 2$ helix (Fig. 12c-d). These results are also in line with the fluctuations of $\alpha 2$ in the per residue RMSD analysis of DM1-SIM1 (Fig. S3). Additionally, the junction 23 (J23) connecting $\alpha 2$ and $\alpha 3$ is highly mobile in DM1-SIM1. In wild type and DM2 simulations, however, movement of J45 connecting $\alpha 4$ and $\alpha 5$ (junction of $\alpha 4-\alpha 5$ ) was observed (Figs. S13-S15).

In general, DM1-SIM1 displays remarkably higher fluctuations and scans different conformational spaces. Although the global dynamics of DM2-SIM1 are similar to WT simulations, 2 out of 3 DM2 simulations behave different from the wild-type, implying change in structure and essential dynamics of the binding groove upon deamidation (Fig. S15). In the deamidated systems, particularly DM1, the binding groove motions are significantly different from the rest, suggesting prominent impact of IDR deamidation on the distal BG. It should be noted that some motions in the binding groove (helices or junctions) seem partly similar for all systems (WT, DM1, and DM2), but overall motions of corresponding PCs do not overlap among different systems. The results suggest that PC motions are complex, regardless of the 
identity of the system. Moreover, these outcomes emphasize the importance of performing multiple and microsecond long simulations to observe the full spectrum of possible motions. Previously, in their computational study, Priya et al. reported that while $\mathrm{Bcl}-\mathrm{X}_{\mathrm{L}}$ without IDR displayed open-close motions (PDB: 1R2DX-Ray, residues 2-29, 83-196), Bcl-XL with IDR (PDB:1LXLNMR, residues 2-196) showed swinging motions, correlating with the longitudinal motions of $\alpha 2$ and $\alpha 3$ during $100 \mathrm{~ns} \times 2 \mathrm{MD}$ simulations. ${ }^{53}$ Contrary to Priya's results, we observed open-close motions in all systems that bear the IDR (Fig. 12, Figs. S13-S15). This result emphasizes the importance of long simulation time and more importantly, the necessity to use of the complete protein structure, which includes the IDR.
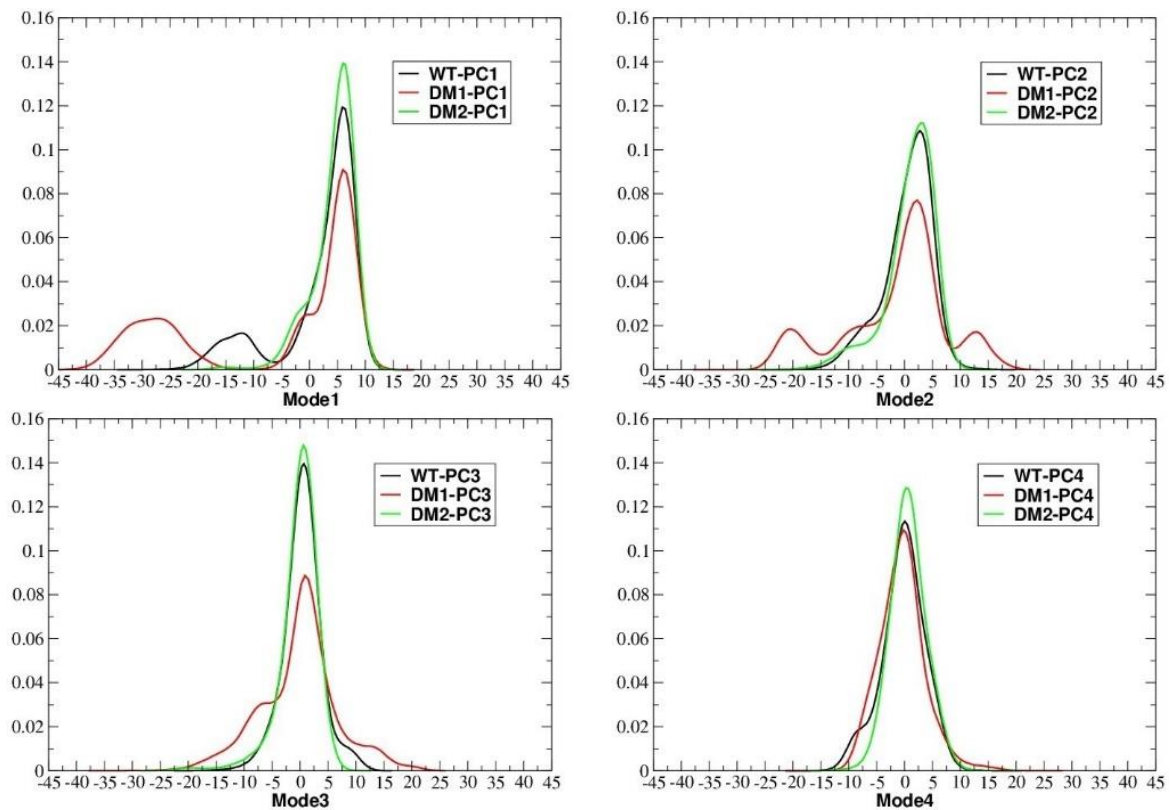

Fig. 9. Top four PC projections (modes) for each system (WT, DM1, and DM2). (Zero (0) indicates the average structure.)
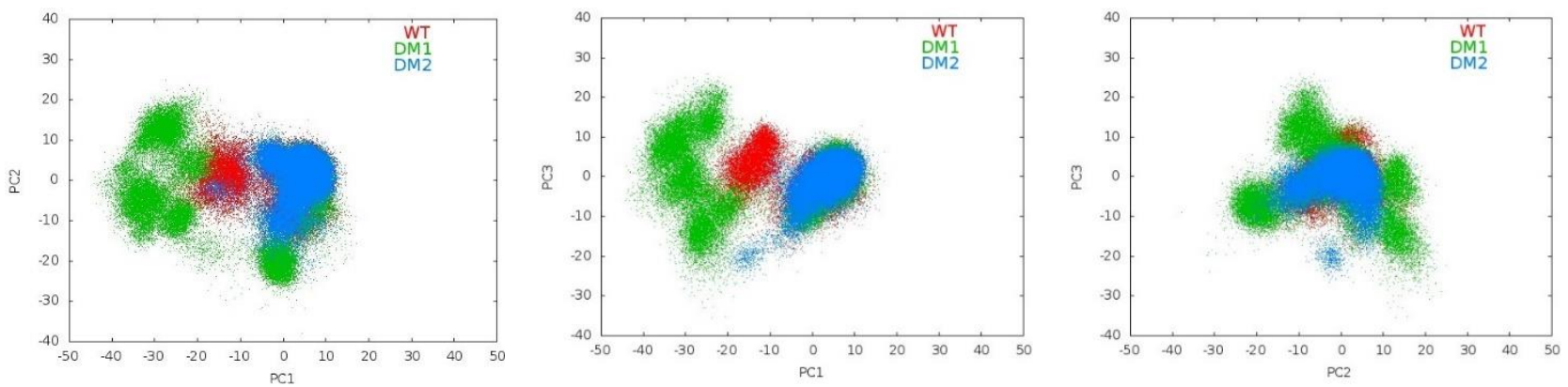

Fig. 10. Projection of top three PC modes onto the combined MD runs (WT+DM1+DM2). (Every 10 points were plotted.)

Taken together, PCA findings revealed that PC motions of DM1 and DM2 considerably differ from WT and suggest that deamidation impacts the structure and motion of $\mathrm{Bcl}-\mathrm{x}_{\mathrm{L}}$, in particular, the dynamics surrounding the BG. This, in turn, may lead to loss of ability to bind $\mathrm{BOP} /$ pro-apoptotics, rendering BCl-XL unfit for its anti-apoptotic function. Particularly, DM1 
deamidation in the IDR causes remarkable changes in the essential motions of the $\mathrm{BCl}-\mathrm{x}_{\mathrm{L}}$ binding groove. Based on these outcomes, it could be concluded that deamidation of IDR allosterically influences the binding groove by changing the dynamics of the protein. This finding conforms with Follis's ${ }^{40}$ suggestion that PTMs on the IDR may allosterically remodel the binding groove to trigger apoptosis. Furthermore, MD results also show the pivotal role of performing multiple simulation to understand the impact of deamidations and to explore the behavior of the studied systems more efficiently.

\section{Impact of Deamidation on the Binding Groove (BG)}

Significant differences in essential dynamics (via PC modes) of DM1 encouraged us to further explore the impact of deamidation on the binding groove (BG) by studying more closely the interaction patterns, specifically $\mathrm{H}$-bonding. We observed that in DM1-SIM1 the junction 23 connecting $\alpha 2$ and $\alpha 3$ (J23) moves towards both the $\alpha 4$ helix and the junction of $\alpha 4$ and $\alpha 5$ (J45), that is, causing the narrowing of the binding groove (Fig. 13). The distance plots for the full trajectories are given in Fig. S16. In order to pinpoint the residues that contribute to the narrowing of the binding groove, hydrogen bonds ( $\mathrm{H}$-bond) between residues lying on either side of the BG were explored and the interactions that are either persistent (long-lived) or short-lived but re-occur in more than $30 \%$ of the MD simulations were discussed herein.

Priya et al. also reported that R103 (J23) and R139 ( $\alpha 5)$ of wild-type Bcl-xL behave like a gate by covering the bottom side of the binding groove compared to that of $\mathrm{BCl}-\mathrm{X}_{\mathrm{L}}$ without IDR. ${ }^{53}$ We observed this type of behavior in DM1-SIM1, alas, between different residues. Most prominently, in DM1-SIM1, R102 on $\mathrm{J} 23$ persistently interacts with D133 (J45) (77.1\%) and E129 ( $\alpha 4$ ) (45.8 \%) indicating the importance of the R102 residue (Fig. 14a). Indeed, R102 is primarily responsible for the narrowing of the binding groove. However, these interactions are absent in all other simulations, except WT-SIM4 (R102-D133 = 31.9 \%, R102-E129 = 14.9 $\%)$. Instead, R102 mostly interacts with E98 and S106 in all other simulations, regardless of the system (Fig. 14c). The R102-E98 and R102-S106 interactions move R102 away from the groove and cause the bottom side (J23 and J45) of the groove to stay open (Fig. 15a). Another important residue involved in the narrowing of the bottom side of the groove (J23-J45) is E129 ( $\alpha 4)$, which interacts with Y101 (J23) (34.0\%) and R103 (J23) (34.1 \%) (Fig. 14a-b). 

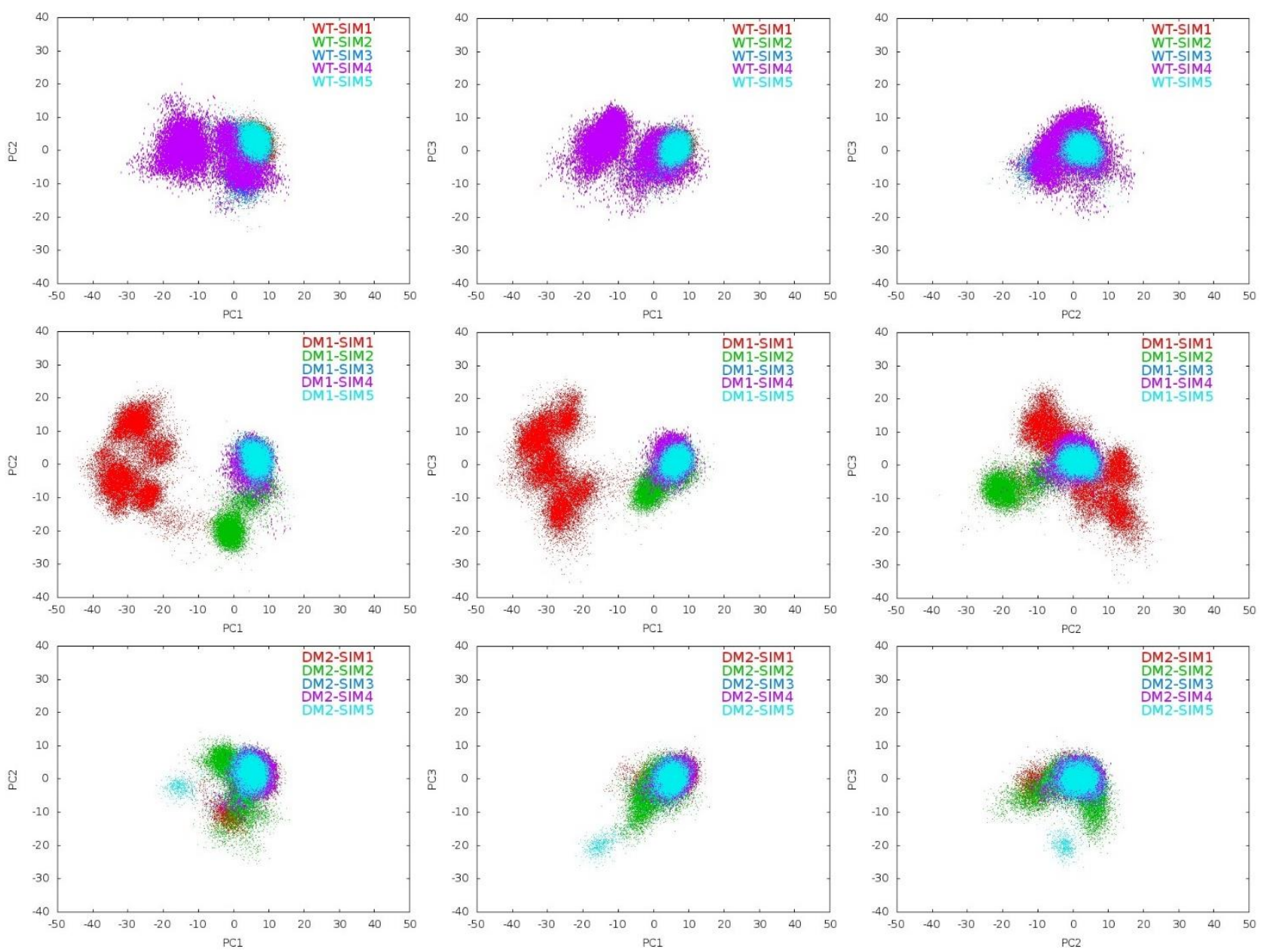

Fig. 11. PC scatter plots for top three PC modes of WT (top), DM1 (middle), DM2 (bottom) MD runs onto all MD runs. (Every 10 points were plotted.)

Moreover, in DM1-SIM1 H-bond interactions between R103 (J23) and Q111 ( $\alpha 3$ ), (70.0 \%) and between E98 (J23), and F105 ( $\alpha 3),(47.6 \%)$ were observed, which result in the covering of the top of the groove (Figs. 14b and 15b). In WT-SIM4 a slight narrowing of the binding groove was also detected, but was less prominent compared to the DM1-SIM1 case (Figs. S16-S17). Lastly, in 2019, Lee and Fairlie pointed out notable differences in the orientation of Phe105 ( $\alpha 3$ ) and Tyr101 (J23) among $\mathrm{Bcl}-\mathrm{X}_{\mathrm{L}}$ complex structures depending on the identity of the $\mathrm{BOP}^{72}$ and pro-apoptotic peptides it engaged. ${ }^{28}$ It should be noted that these two residues play a pivotal role in binding to BOPs and pro-apoptotics. ${ }^{72,73}$ Remarkably, we observed that the Tyr101-Phe105 interaction is almost nonexistent in DM1-SIM1 and DM1-SIM2 simulations, while persistent in all other simulations, indicating that deamidation has led to the disruption of a significant interaction enabling Bcl-x's's binding to proapoptotic peptides. This in turn leads to its loss of anti-apoptotic function. Taken together, asparagine deamidation to aspartate in the IDR seems to allosterically induce the binding groove from an "open" to a "narrow" state. More specifically, the rotations and interactions of $J 23$ and $\alpha 3$ residues, particularly R102 and R103 have a significant effect on the rearrangement of the binding groove (Figs. 14 and 15). 


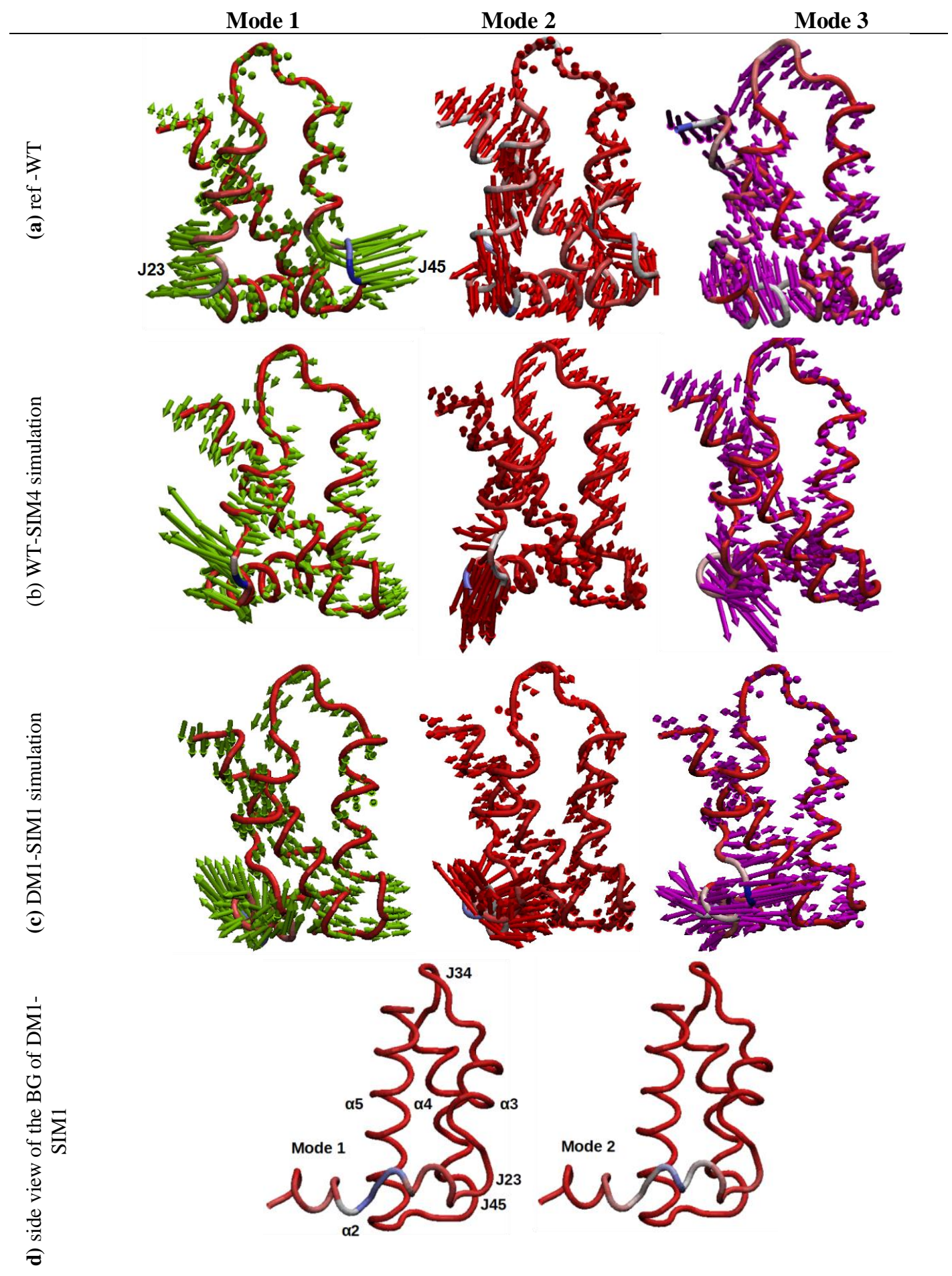

Fig. 12. The first three PC modes for (a) ref-WT (combination of simulations WT-SIM1, 2 and 5), (b) WT-SIM4 and (c) DM1-SIM1. (d) Side view of the BG in DM1-SIM1. (Blue color indicates higher residue mobility. Arrows display the direction and magnitude of the motion.) 


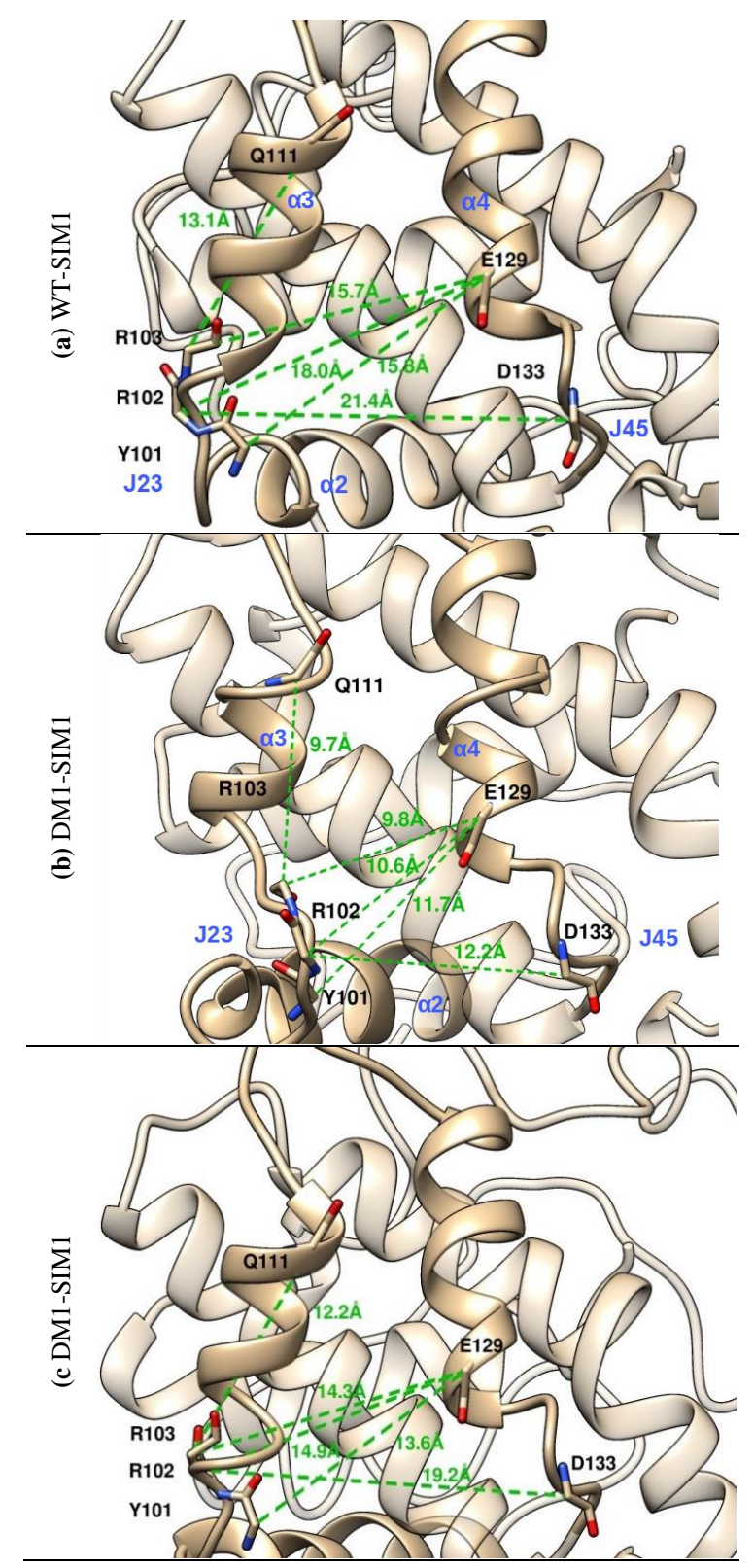

Fig. 13. Representative snapshots and $\mathrm{C} \alpha$ distances of key residues at the BGs of WT-SIM1, DM1SIM1 and DM2-SIM1. Snapshots taken at $5 \mu \mathrm{s}$. 


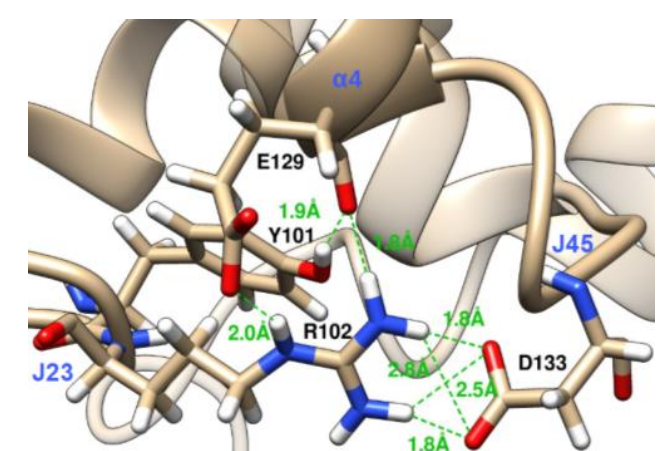

(a) R102-D133, R102-E129, Y101-E129 in DM1-SIM1

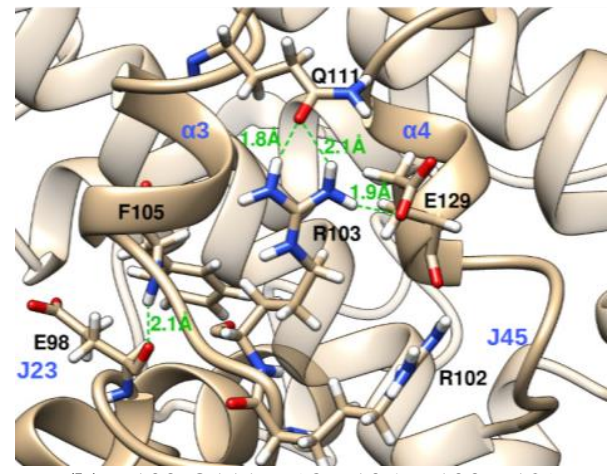

(b) R103-Q111, E98-F105, R103-E129 in DM1-SIM1

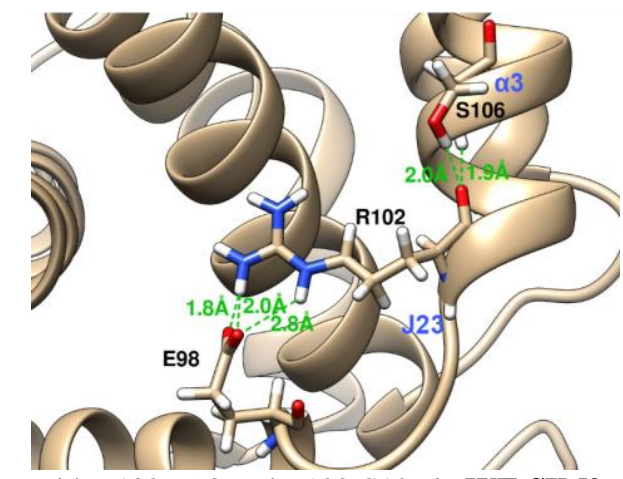

(c) R102-E98 and R102-S126 in WT-SIM2

Fig. 14. Representation of key $\mathrm{H}$-bond interactions between $\mathrm{BG}$ residues that are short-lived and consistently re-occur in more than $30 \%$ of the simulation time a-b) DM1-SIM1, and c) WT-SIM2.

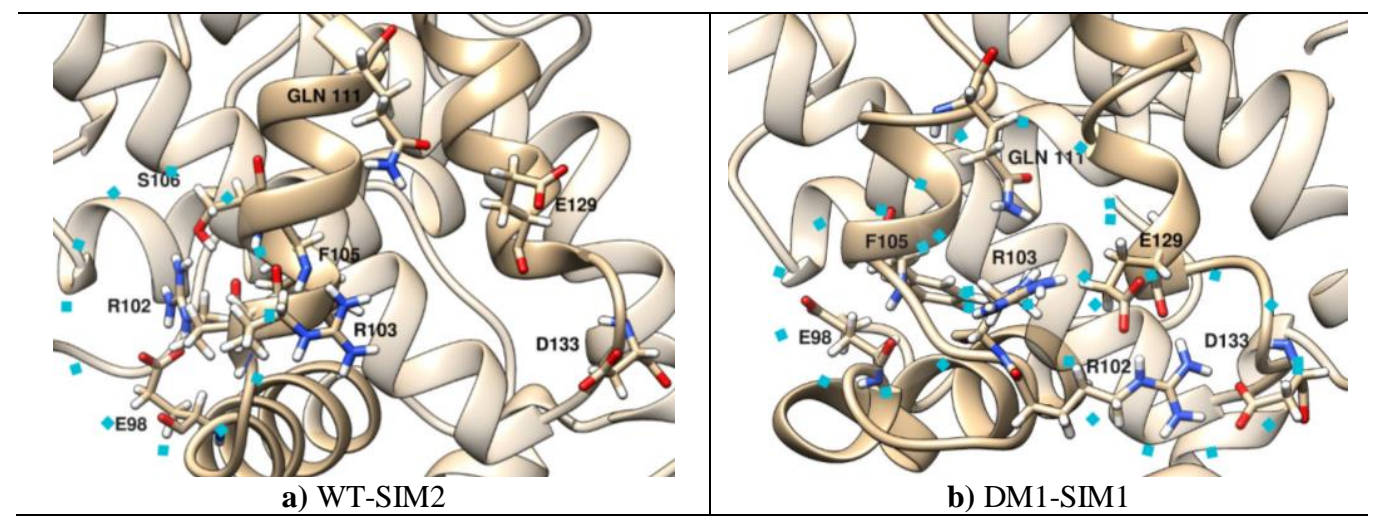

Fig. 15. Orientation of the key BG residues in a) WT-SIM2 and b) DM1-SIM1. 


\section{Conclusions}

This study entails a significant contribution to the knowledge on structural consequences of deamidation on $\mathrm{Bcl}-\mathrm{x}_{\mathrm{L}}$. The increase in negatively charged residues on the IDR, as a result of deamidation, was shown to change the essential dynamics of the binding groove of $\mathrm{BCl}-\mathrm{x} \mathrm{L}$ and hence, to alter its structural behavior and conceivably impair its function. One of the most important findings of this study is that the change in IDR (loop) motions upon deamidation may significantly affect accessibility of the binding groove. Indeed, the IDR allosterically influences the BG of the protein and induces conformational modifications that lead to changes in the interaction network. Our findings suggest that deamidation in the IDR changes both the number (\%) and type of non-bonded interactions between the IDR and Bcl-xL's core. The change in BG interactions occur mainly in subunits $J 23, \alpha 3, \alpha 4$, and J45, and include five specifics, previously non-existent, interactions, namely, R102-D133 and R102-E129, E129Y101 and E129-R103, and R103-Q111. These allosterically induced interactions are shown to be responsible for the narrowing and covering of the groove upon deamidation. Moreover, PCA analysis along the first three principal components show remarkable differences in essential motions of the binding groove's of wild type and deamidated Bcl-XL. These outcomes suggest that deamidation on the IDR not only impacts the structure by causing remarkable changes in the essential motions of the binding groove but alters its structural behavior and expectedly impairs its function.

These findings will bring a unique perspective to the underlying mechanism of $\mathrm{Bcl}-\mathrm{X}_{\mathrm{L}}$ deamidation-induced cell death by bringing forward the structural knowledge necessary to develop anti-cancer therapeutics. Additionally, a concerted effort is required and underway to fully explore the structural changes that full-length $\mathrm{Bcl}-\mathrm{XL}_{\mathrm{L}}$ (including both IDR and C-tail) undergoes upon PTMs and the consequences on its complex biological environment, including the dynamics of the mitochondrial outer membrane and its specific protein-protein interactions. This study is a first step in understanding the effect of deamidation on the structure and function of $\mathrm{Bcl}-\mathrm{xL}_{\mathrm{L}}$ by using a non-truncated and unmodified model, also highlighting the importance of longer and multiple simulations.

\section{Supporting Information}

The following files are available free of charge.

Force field parameterization of non-standard iso-Asp, per residue RMSD and secondary structure analyses, B-factor coloring and radial distribution function plots, principal component analysis, and distance plots. (PDF)

\section{Acknowledgements}

GT and SC acknowledge TUBITAK and Bogazici University Research Fund (BAP-M) for financial support under grant number $116 Z 513$ and 14260, respectively. GT thanks the French Embassy in Ankara, Turkey, for the co-tutelle grant in France. GPU resources were allocated by the Mésocentre EXPLOR of the Université de Lorraine and the Centre de Cal-cul ROMEO of the Université de Reims Champagne-Ardenne as well as TUBITAK ULAKBIM High Performance and Grid Computing Center (TRUBA resources). 


\section{References}

A. Schinzel, T. Kaufmann and C. Borner, Biochim. Biophys. Acta - Mol. Cell Res., 2004, 1644, 95-105.

R. Singh, A. Letai and K. Sarosiek, Nat. Rev. Mol. Cell Biol., 2019, 20, 175-193.

P. E. Czabotar, G. Lessene, A. Strasser and J. M. Adams, Nat. Rev. Mol. Cell Biol., 2014, 15, 49-63.

S. Cory and J. M. Adams, Nat. Rev. Cancer, 2002, 2, 647-656.

S. H. Leech, R. A. Olie, O. Gautschi, A. P. Simões-Wüst, S. Tschopp, R. Häner, J. Hall, R. A. Stahel and U. Zangemeister-Wittke, Int. J. cancer, 2000, 86, 570-6.

T. Takehara and H. Takahashi, Cancer Res., 2003, 63, 3054-7.

R. Zhao, G. A. Follows, P. A. Beer, L. M. Scott, B. J. P. Huntly, A. R. Green and D. R. Alexander, N. Engl. J. Med., 2008, 359, 2778-2789.

G. Walsh and R. Jefferis, Nat. Biotechnol., 2006, 24, 1241-1252.

G. Walsh, Drug Discov. Today, 2010, 15, 773-780.

Z.-J. Han, Y.-H. Feng, B.-H. Gu, Y.-M. Li and H. Chen, Int. J. Oncol., 2018, 52, 1081-1094.

N. E. E. Robinson, Z. W. W. Robinson, B. R. R. Robinson, A. B. B. A. L. L. Robinson, J. A. A. Robinson, M. L. L. Robinson and A.

B. B. A. L. L. Robinson, J. Pept. Res., 2004, 63, 426-436.

H. Tonie Wright and D. W. Urry, Crit. Rev. Biochem. Mol. Biol., 1991, 26, 1-52.

T. Geiger and S. Clarke, J. Biol. Chem., 1987, 262, 785-794.

N. E. Robinson and A. B. Robinson, Proc. Natl. Acad. Sci. U. S. A., 2001, 98, 944-9.

S. Catak, G. Monard, V. Aviyente and M. F. Ruiz-López, J. Phys. Chem. A, 2006, 110, 8354-8365.

M. Aritomi, N. Kunishima, N. Inohara, Y. Ishibashi, S. Ohta and K. Morikawa, J. Biol. Chem., 1997, 272, 27886-27892.

S. W. Muchmore, M. Sattler, H. Liang, R. P. Meadows, J. E. Harlan, H. S. Yoon, D. Nettesheim, B. S. Chang, C. B. Thompson, S.-L. Wong, S.-C. Ng and S. W. Fesik, Nature, 1996, 381, 335-341.

L. H. Boise, M. González-García, C. E. Postema, L. Ding, T. Lindsten, L. A. Turka, X. Mao, G. Nuñez and C. B. Thompson, Cell, 1993, 74, 597-608.

E. H. Y. Cheng, M. C. Wei, S. Weiler, R. A. Flavell, T. W. Mak, T. Lindsten and S. J. Korsmeyer, Mol. Cell, 2001, 8, 705-711.

B. Leber, J. Lin and D. W. Andrews, Oncogene, 2010, 29, 5221-5230.

C. Borrás, C. Mas-Bargues, A. Román-Domínguez, J. Sanz-Ros, L. Gimeno-Mallench, M. Inglés, J. Gambini and J. Viña, Int. J. Mol. Sci., 2020, 21, 418.

B. Levine, S. C. Sinha and G. Kroemer, Autophagy, 2008, 4, 600-606.

E. F. Lee, N. A. Smith, T. P. Soares da Costa, N. Meftahi, S. Yao, T. J. Harris, S. Tran, A. Pettikiriarachchi, M. A. Perugini, D. W. Keizer, M. Evangelista, B. J. Smith and W. D. Fairlie, Autophagy, 2019, 15, 785-795.

C. Borras, K. M. Abdelaziz, J. Gambini, E. Serna, M. Inglés, M. de la Fuente, I. Garcia, A. Matheu, P. Sanchís, A. Belenguer, A. Errigo, J. A. Avellana, A. Barettino, C. Lloret-Fernández, N. Flames, G. Pes, L. Rodriguez-Mañas and J. Viña, Aging (Albany. NY)., 2016, 8, 3185-3208.

R. Yosef, N. Pilpel, R. Tokarsky-Amiel, A. Biran, Y. Ovadya, S. Cohen, E. Vadai, L. Dassa, E. Shahar, R. Condiotti, I. Ben-Porath and V. Krizhanovsky, Nat. Commun., 2016, 7, 11190.

J. M. Vicencio, L. Galluzzi, N. Tajeddine, C. Ortiz, A. Criollo, E. Tasdemir, E. Morselli, A. Ben Younes, M. C. Maiuri, S. Lavandero and G. Kroemer, Gerontology, 2008, 54, 92-99.

Y. Yao, D. Nisan, A. Antignani, A. Barnes, N. Tjandra, R. J. Youle, F. M. Marassi and L. M. Fujimoto, Protein Expr. Purif., 2016, 122, 56-63.

E. F. Lee and W. D. Fairlie, Int. J. Mol. Sci., 2019, 20, 2234.

K. Raltchev, J. Pipercevic and F. Hagn, Chem. - A Eur. J., 2018, 24, 5493-5499.

X. Liu, Z. Jia, J. Chen, J. Phys and B. Chem, J. Phys. Chem. B, 2017, 121, 9160-9168.

X. Liu, A. Beugelsdijk and J. Chen, Biophys. J., 2015, 109, 1049-1067.

A. Maity, S. Yadav, C. S. Verma and S. Ghosh Dastidar, PLoS One, 2013, 8, e76837.

R. B. Hill, K. R. MacKenzie and M. C. Harwig, J. Mol. Biol., 2015, 427, 2257-2261.

D. Lama and R. Sankararamakrishnan, Proteins Struct. Funct. Genet., 2008, 73, 492-514.

B. S. Chang, A. J. Minn, S. W. Muchmore, S. W. Fesik and C. B. Thompson, EMBO J., 1997, 16, 968-977.

S. Kharbanda, S. Saxena, K. Yoshida, P. Pandey, M. Kaneki, Q. Wang, K. Cheng, Y. N. Chen, A. Campbell, T. Sudha, Z. M. Yuan, J. Narula, R. Weichselbaum, C. Nalin and D. Kufe, J. Biol. Chem., 2000, 275, 322-327.

J. H. Zheng, A. Viacava Follis, R. W. Kriwacki and T. Moldoveanu, FEBS J., 2016, 283, 2690-2700.

R. J. Clem, E. H. Cheng, C. L. Karp, D. G. Kirsch, K. Ueno, A. Takahashi, M. B. Kastan, D. E. Griffin, W. C. Earnshaw, M. A. Veliuona and J. M. Hardwick, Proc. Natl. Acad. Sci. U. S. A., 1998, 95, 554-559.

G. Basañez, J. Zhang, B. N. Chau, G. I. Maksaev, V. A. Frolov, T. A. Brandt, J. Burch, J. M. Hardwick and J. Zimmerberg, J. Biol. 
Chem., 2001, 276, 31083-31091.

A. V. Follis, F. Llambi, H. Kalkavan, Y. Yao, A. H. Phillips, C.-G. Park, F. M. Marassi, D. R. Green and R. W. Kriwacki, Nat. Chem. Biol., 2018, 14, 458-465.

F. Beaumatin, M. El Dhaybi, C. Bobo, M. Verdier and M. Priault, Biochim. Biophys. Acta-Mol. Cell Res., 2017, 1864, 17341745.

A. Letai and O. Kutuk, Curr. Mol. Med., 2008, 8, 102-118.

J. Kale, E. J. Osterlund and D. W. Andrews, Cell Death Differ., 2017, 25186, 65-80.

Y. Yao, L. M. Fujimoto, N. Hirshman, A. A. Bobkov, A. Antignani, R. J. Youle and F. M. Marassi, J. Mol. Biol., 2015, 427, 22622270.

B. E. Deverman, B. L. Cook, S. R. Manson, R. A. Niederhoff, E. M. Langer, I. Rosová, L. A. Kulans, X. Fu, J. S. Weinberg, J. W. Heinecke, K. A. Roth and S. J. Weintraub, Cell, 2002, 111, 51-62.

R. Zhao, D. Oxley, T. S. Smith, G. A. Follows, A. R. Green and D. R. Alexander, PLoS Biol., 2007, 5, 0039-0053.

S. H. Dho, B. E. Deverman, C. Lapid, S. R. Manson, L. Gan, J. J. Riehm, R. Aurora, K.-S. S. Kwon and S. J. Weintraub, PLoS Biol., 2013, 11, 12201901-14924.

S. H. Dho, S. R. Manson, S. H. Jung, J. C. Lim and S. J. Weintraub, Biochim. Biophys. Acta - Mol. Cell Res., 2018, 1865, 9951001.

B. E. Deverman, B. L. Cook, S. R. Manson, R. A. Niederhoff, E. M. Langer, I. Rosová, L. A. Kulans, X. Fu, J. S. Weinberg, J. W. Heinecke, K. A. Roth and S. J. Weintraub, Cell, 2003, 115, 503.

J. S. Ahn, J. Li, E. Chen, D. G. Kent, H. J. Park and A. R. Green, Oncogene, 2016, 35, 2235-2246.

M. Fares, M. Abedi-Valugerdi, M. Hassan and Z. Potácová, Biochem. Biophys. Res. Commun., 2015, 463, $268-274$.

T. Asakura, K. Maeda, H. Omi, H. Matsudaira and K. Ohkawa, Int. J. Oncol., 2008, 33, 389-395.

P. Priya, A. Maity and S. Ghosh Dastidar, Proteins Struct. Funct. Bioinforma., 2017, 85, 1567-1579.

P. Ryzhov, Y. Tian, Y. Yao, A. A. Bobkov, W. Im and F. M. Marassi, Biophys. J., 2020, 119, 1324-1334.

D. A. Case, S. R. Brozell, D. S. Cerutti, I. T. E. Cheatham, V. W. D. Cruzeiro, T. A. Darden, R. E. Duke, D. Ghoreishi, H. Gohlke, A. W. Goetz, D. Greene, R. Harris, N. Homeyer, S. Izadi, A. Kovalenko, T. S. Lee, S. LeGrand, P. Li and P. A. Lin, University of California: San Francisco, 2018.

J. A. Maier, C. Martinez, K. Kasavajhala, L. Wickstrom, K. E. Hauser and C. Simmerling, J. Chem. Theory Comput., 2015, 11, 3696-3713.

W. L. Jorgensen, J. Chandrasekhar, J. D. Madura, R. W. Impey and M. L. Klein, J. Chem. Phys., 1983, 79, 926-935.

H. C. Andersen, J. Chem. Phys., 1980, 72, 2384-2393.

U. Essmann, L. Perera, M. L. Berkowitz, T. Darden, H. Lee and L. G. Pedersen, J. Chem. Phys., 1995, 103, 8577-8593.

J. P. Ryckaert, G. Ciccotti and H. J. C. Berendsen, J. Comput. Phys., 1977, 23, 327-341.

H. J. C. Berendsen, J. P. M. Postma, W. F. van Gunsteren, A. DiNola and J. R. Haak, J. Chem. Phys., 1984, 81, 3684-3690.

J. A. Izaguirre, D. P. Catarello, J. M. Wozniak and R. D. Skeel, J. Chem. Phys., 2001, 114, 2090-2098.

C. W. Hopkins, S. Le Grand, R. C. Walker and A. E. Roitberg, J. Chem. Theory Comput., 2015, 11, 1864-1874.

D. R. Roe and T. E. Cheatham, J. Chem. Theory Comput., 2013, 9, 3084-3095.

W. Kabsch and C. Sander, Biopolymers, 1983, 22, 2577-2637.

A. Bakan, L. M. Meireles and I. Bahar, Bioinformatics, 2011, 27, 1575-1577.

E. F. Pettersen, T. D. Goddard, C. C. Huang, G. S. Couch, D. M. Greenblatt, E. C. Meng and T. E. Ferrin, J. Comput. Chem., 2004, 25, 1605-1612.

T. D. Goddard, C. C. Huang, E. C. Meng, E. F. Pettersen, G. S. Couch, J. H. Morris and T. E. Ferrin, Protein Sci., 2018, 27, 14-25. W. Humphrey, A. Dalke and K. Schulten, J. Mol. Graph., 1996, 14, 33-38.

C. Bobo, C. Céré, M. Dufossée, A. Dautant, V. Moreau, S. Manon, F. Beaumatin and M. Priault, Int. J. Mol. Sci., 2019, 20, 5571.

A. Amadei, A. B. M. Linssen and H. J. C. Berendsen, Proteins Struct. Funct. Bioinforma., 1993, 17, 412-425.

S. Rajan, M. Choi, K. Baek and H. S. Yoon, Proteins Struct. Funct. Bioinforma., 2015, 83, 1262-1272.

A. Priyadarshi, A. Roy, K. S. Kim, E. E. K. Kim and K. Y. Hwang, Biochem. Biophys. Res. Commun., 2010, 394, 515-521. 